\title{
INCENTIVE DESIGN WITH CUSTOMER SATISFACTION FOR BUSINESS PROCESS OUTSOURCING: MULTI-TASK VS. MULTI-AGENT
}

\author{
Zhenyang Shi ${ }^{1}$, Shaoxuan LiU ${ }^{1,2}$ And Ruijing Wu ${ }^{1, *}$
}

\begin{abstract}
We study the incentive design decision of a firm that outsources its online marketing and operational business lines to professional external and internal service providers under the principalagent framework. Customer satisfaction is represented as the composite of the agents' sales and qualitycontrol efforts via the disconfirmation model, and is introduced into the demand model and incentive contracts. Two scenarios corresponding to whether the brand owner delegates business to a single agent or to multiple agents are studied. We derive the brand owner's optimal contracts and the agents' effort levels and conduct extensive sensitivity analyses regarding the influences of model parameters. We find that increases in the measure accuracy of customer satisfaction can bring significant benefit to the firm's profitability, and explore how it is affected by system factors. Specifically, the benefit is more substantial when the marginal cost of quality-control effort is relatively smaller than the marginal cost of sales effort. An interesting discovery is that the brand owner always earns more profit by outsourcing business to multiple agents than to a single agent, mainly because of his inflexibility in reaching a balance between inducing higher effort levels and providing a sufficient risk premium in the singe-agent case. This profit gap can be expanded by incentivizing both agents on customer satisfaction measures, and is shown quite significant under certain settings in the numerical studies.
\end{abstract}

Mathematics Subject Classification. 91A80, 91A40, 90B60.

Received January 22, 2019. Accepted June 7, 2019.

\section{INTRODUCTION}

With the ever-increasing popularity of e-commerce, manufacturers and retailers have realized the importance of online channels as a profitable complement to traditional channels. However, due to their inexperience in conducting e-business, many firms outsource their online marketing business to professional service agents who provide various services, such as online stores design, advertisement campaigns and promotion on social network sites. For example, several business-to-business platforms for online marketing service have emerged in China, including Siilu.com and Taobao Service. Another common observation in recent years is that, to focus on their core competencies, many firms in industries such as apparel and consumer electronics have outsourced their operational business, like procurement, manufacturing, and quality control, to contract manufacturers (CM),

Keywords. Game theory, incentive design, customer satisfaction, supply chain management, principal-agent models.

1 Antai College of Economics and Management, Shanghai Jiao Tong University, 200030 Shanghai, PR China.

2 Ningbo Supply Chain Innovation Institute China, MIT Global Supply Chain and Logistics Excellence (SCALE) Network, 315000 Ningbo, PR China.

*Corresponding author: wrj9128@sjtu.edu.cn 
while becoming brand owners themselves [57]. In the electronics industry, Foxconn is a typical CM providing a wide range of operational services to its OEMs such as Apple and HP [62]. Another example is the emergence of online marketplaces that enables manufacturing outsourcing, such as Alibaba's Tao Factory, on which retailers can procure goods from their manufacturing partners [60]. Although outsourcing has its cost advantage, it is also risky for firms because of the more difficulties in observing the outside companies' actual efforts, such as the quality improvement and maintenance activities of CMs $[25,34,48]$. Therefore, the moral hazard phenomenon arises, and it is critical for firms to devise effective incentive contracts to motivate the outside partners (marketing service providers and $\mathrm{CMs}$ ) to align their interests with those of the firm itself.

The above practices belong to the business process outsourcing (BPO) model, where a firm's business functions are carried out by its outside partners. In practice, to focus on the online business or a specific niche market, firms may establish independent subsidiaries with several functional departments such as marketing and operations. For example, NetEase, a Chinese Internet company, created its own e-commerce brand, Yanxuan, which operates under the original design manufacturer (ODM) model. The business unit has its departments like supply chain, marketing, customer service, and is featured with high involvement in upstream supply chain operations, especially supplier selection and quality control [40,43]. This business model, which has also been adopted by phone maker Xiaomi that operates its own retail brand Youpin [61], is indeed an "intra-organizational outsourcing" model, because the business unit has autonomy in decisions and acts as a "multitasking agent" $[18,19]$. Another similar business model is franchising, which allows the franchisees to have considerable freedom for independent decision [45], and has been widely adopted in many industries, e.g., hotels and car rentals [24]. In such settings, it is also imperative for firms to effectively motivate their subsidiaries to carry out various job functions and improve profitability by appropriate compensation plans.

Customer satisfaction (CS) measures how products and services meet and surpass customers' expectations and is closely related to companies' long-run profitability. A mainstream CS quantitative model, the disconfirmation model, states that CS is a function of customers' post-purchase perceived quality and the difference (disconfirmation) of perceived and expected quality [2]. Note that perceived quality is affected mainly by quality improvement and control effort, while expectations are affected by customers' pre-purchase evaluation resulting from marketing-related activities, e.g., sales effort. Therefore, CS can be an appropriate proxy to measure the invisible quality-control and sales efforts in moral hazard phenomena. In fact, CS has been employed to incentivize employees for quite a long time [26], and CS-based compensation is adopted in industries including investment [23], banking [7], and healthcare [31]. However, the extant research views CS as the result of a single-dimension effort and rarely considers incentive design problem including CS with its formulation based on the disconfirmation model. Therefore, it is interesting to investigate how a transfer payment incorporating CS is optimally designed by firms in the BPO scenarios above. To measure CS value in the e-business environment, in addition to traditional approaches such as CS surveys through phone calls or e-mails, firms can utilize customers' online ratings and feedback as a new information source. Although more data on CS are currently available at a lower cost, they may include biased or irrelevant information, which makes the extraction of true CS value more difficult. Ho et al. [29] reveal that a product's online ratings may not neutrally reflect customers' post-purchase evaluation due to the disconfirmation effect. Therefore, it is also intriguing to explore how the accuracy of CS measurement can affect the incentive design decision.

Motivated by the phenomena above, this paper focuses on a brand owner that delegates its online marketing and operational (especially product manufacturing and quality control) business to (external or internal) professionals. The business models mentioned above, e.g., a firm outsources its business lines to separate service providers, or lets a independent subsidiary take control of the business functions, are both considered. In this paper we refer to them as "outsourcing models", regardless of whether they correspond to an inter-firm or an intra-firm delegation relationship. Thus "outsourcing" in this paper is equivalent to "delegation", and also includes the case where a firm's own business units are in charge of its marketing and production functions. We assume that sales effort determines the firm's current demand, while future demand is affected by CS, modeled as a composite of both sales and quality-control efforts according to the disconfirmation model: Quality-control effort promotes CS whereas sales effort negatively impacts CS. We study the incentive design 
problem under the principal-agent (PA) framework and investigate both the multi-task case and multi-agent case, which respectively correspond to the two outsourcing models above. Optimal incentive plans are derived and extensive sensitivity analyses on the impacts of various parameters on the optimal solutions are conducted, alongside a comparative analysis of the results under the two scenarios. The subsection below provides a comprehensive review of relevant literature, where we discuss the similarities of our paper to the extant research, and emphasize our distinctive contributions regarding problem formulation and major findings.

\subsection{Related literature and contributions}

Our paper is closely related to three streams of literature, i.e., research works on customer satisfaction, salesforce compensation design, and marketing-operations interface.

The disconfirmation model of CS has been constructed and validated by Oliver [49], Bolton and Drew [6] and Cronin Jr and Taylor [13]. Anderson and Sullivan [2] formulate CS with an additive form composed of perceived quality and disconfirmation. Several papers incorporate this model into analytical frameworks on the relationship between CS and firm profitability. Ho et al. [28] connect CS with customer purchase rate, carry out a customer lifetime value analysis based on CS and derive an optimal investment decision. Kopalle and Lehmann [35] consider a two-period problem in which CS is explicitly expressed as the disconfirmation affected by advertisement and product quality, and emphasize its impact on future demand. Aflaki and Popescu [1] construct the evolution of CS as an adaptive expectation process depending on past service levels. By linking CS with customer retention, they formulate a dynamic program and derive the optimal long-run policy. All these papers consider the centralized decision-making scenario. Our paper differs by considering a similar formulation of CS under a decentralized environment where contracting issues between different parties are concerned.

Since the seminal paper by Basu et al. [3], the research field on salesforce compensation design has been well developed, with various factors studied, such as asymmetric information [37,39], multi-product scenarios [38], pricing decision delegation $[4,46]$, dynamic environment $[54,56]$. Readers are referred to Misra et al. [47] and Rouziès et al. [53] for a more detailed review. These research works rarely incorporate CS-related indicators into the compensation plan. Kalra et al. [26] first consider CS measure as an incentive term, where the firm compensates its employees on both sales and CS to mitigate their myopia in allocating effort under a two-period setting, assuming that CS is affected by the enduring effort that determines future demand. Kalra et al. [33] study a the scenario in which products are technically complicated and salespersons may overstate the value of added features. They find that by including CS measure into compensation, firms can signal to customers the actual value of an upgrade feature and mitigate the overselling phenomenon. Xiao et al. [63] consider a supply chain in which a risk-averse manufacturer incentivizes a risk-averse retailer's short-term selling effort, customersatisfying effort and pricing decision. They identify the impacts of risk sensitivity and demand uncertainty on the equilibrium results. Deng et al. [20] demonstrate that CS incentives can benefit firms even in a single-period setting under budget constraints since the CS measure provides additional information on the agent's hidden action and diversifies the risk faced by the agent. All these papers represent CS as a function of a single effort level of the agent, so the disconfirmation model is not employed, and they solve the problem under a single-agent setting. Our paper, however, further incorporates disconfirmation in modeling CS and considers the multi-agent scenario. Although CS is not explicitly mentioned in their paper, Dong et al. [21] consider two kinds of efforts, i.e., customer acquisition and retention, and investigate how their interactions, especially the spoiling effect of acquisition, may affect the optimal effort levels and firm profit under direct selling and delegation. These two kinds of efforts are similar to sales and quality-control effort in our paper, and the spoiling effect resembles the disconfirmation effect. However, in their model setting, Dong et al. [21] compare the results in direct selling and delegation cases with a single agent, while our paper concentrates on both the multi-agent and multi-task scenarios.

Our paper is also related to papers on operations-marketing interfaces [59], especially two streams of literature: Studies on lead time and capacity management in service operations, and compensation design with operational issues. The first stream of research focuses on scenarios where firms can guarantee a certain service level to customers to promote demand, whereas the actual service level is jointly determined by the demand stream 
and firms' operational capacity. The trade-off between service level commitment and capacity decision in this problem is similar to the decision on allocating sales and quality-control efforts in our paper. Ho and Zheng [27] introduce the gap model of service quality in modeling customer's utility under a service system, which is directly from the disconfirmation model above. Readers are referred to So [58], Liu et al. [41], Pekgün et al. [51], Feng et al. [22], etc., for some representative studies in this field.

The second stream of literature is those on salesforce compensation planning integrated with operational decisions. Porteus and Whang [52] consider a firm that contracts with a manufacturing manager responsible for capacity building and with multiple product managers in charge of demand generation and derive a mechanism enabling the first-best result. Chen [8] considers a newsvendor-like firm offering incentives to a salesperson who can observe private information on market conditions, and the author derives the optimal menu of linear contracts. Ma et al. [44] study a two-stage supply chain where demand is jointly determined by the retailer's sales effort and manufacturer's quality improvement effort, and they design a cost sharing contract which enables coordination. More recent research has expanded in several aspects, such as considering demand censorship and double moral hazard [10,15-17], dynamic contract design [55], pricing decisions [9,14,65], etc. Among the existing literature in this stream, two papers $[18,32]$ are closely related to our work. The former paper study a firm under a newsvendor setting, who incentivizes its marketing and operations managers with optimal linear contracts based on product sales and cost reduction. Such a model is similar to the multi-agent scenario in our paper. Dai et al. [18] investigate compensation design for a multitasking store manager who carries out both operations and marketing activities. Their model is featured with the censoring of demand, the outcome of sales effort, by inventory level that increases with operational effort. The optimal contract is characterized by a bonus region with a "mast and sail" structure. The multi-task scenario in our paper shares similarities with this model. The two papers above consider inventory management as the main operational decision, while our paper differs from them by using quality-control effort as the operation-related factor that influences main performance measure, customer satisfaction. Thus our analytical framework resembles that established in literature on incentive design with quality improvement such as Xie et al. [64], Ma et al. [44] and Liu et al. [42]. We focus on the firm's optimal linear contracts designed for the service providers, which is also studied in [32]. Dai et al. [18], on the other hand, identify the optimal compensation plan among all possible candidates, which is a more general problem.

This paper makes several considerable contributions to the existing literature. First, we express the disconfirmation model of CS explicitly with sales and quality-control efforts, and incorporate this formulation into an incentive design problem under both the multi-task and multi-agent scenarios. To the best of our knowledge, studies of these problems are rare in previous research, where the disconfirmation model and CS-based incentives are only studied separately in a single-agent scenario, as the literature review above have shown. Second, through sensitivity analyses, we emphasize the benefit brought by increasing CS measure accuracy. Furthermore, we find that when the marginal cost of quality-control effort is less than that of sales effort, the benefit above can be more significant. The degree of importance of disconfimation in CS determination also influences the profit benefit, whereas the direction and magnitude of the impact is subtle and depends on specific outsourcing models. The results also maintain consistency with the findings in existing literature [21] and provide insightful guidelines for increasing CS measure accuracy in practice. Third, in our comparison of the two outsourcing scenarios, we find that the brand owner always earns more profit under the multi-agent case than the multi-task case, which is somewhat interesting. Through extensive analysis from various perspectives, we conclude that the underlying reason for this difference is that under the multi-task case, the brand owner faces more inflexibility in adjusting incentive decisions to reach a balance between inducing high-level efforts and paying a risk premium to incentivize the agent. This property is essentially related to the disconfirmation model, which is characterized by the opposite effects of the two efforts. Thus the finding reflects the insightful results this model can produce.

The rest of this paper is organized as follows. We introduce some fundamental assumptions and building blocks for the analytical model in Section 2. The PA formulation of our problem is presented, with optimal incentive plans derived and sensitivity analyses of the optimal solutions regarding model parameters carried out for both the multi-task and multi-agent cases in Sections 3 and 4, respectively. In Section 5, we compare 
the two scenarios in terms of profit and provide detailed explanations to facilitate a deep understanding of the differences. We conclude the paper in Section 6. All proofs are delegated to the Appendix A. Throughout the paper, we use positivity/negativity, monotonicity and convexity/concavity in their weak sense.

\section{Model Setup}

Consider a brand owner (henceforth interchangeable with "firm" and "principal") who sells products in online market and outsources both online marketing and operational business lines to professional service providers. The service provider can be either a marketing service provider (MSP) or an operational service provider (OSP) under the multi-agent case, or can be an integrated service provider (ISP) under the multi-task case. In the rest of this paper we will use service provider and agent interchangeably. All decisions are made in a single selling period. The total demand consists of demand in this selling period determined by sales effort and future demand affected by CS in the current period, thus reflecting the dynamic impact of CS. Such assumptions are commonly adopted in the existing literature; see Hauser et al. [26], Chu and Desai [11], Kalra et al. [33], Kopalle and Lehmann [35], among others.

Let the sales effort level be $e_{1}$, which is an abstract representation of marketing-related activities carried out by MSP, and let $D_{1}$ denote current demand. With a higher $e_{1}$, the product will be presented to more potential customers with a higher probability and frequency; thus, customers' expected value for this product will be higher. So without loss of generality, we can view $e_{1}$ as customers' expected value. When $e_{1}$ goes higher, the purchasing probability and, thus, $D_{1}$ will also be higher, which is similar to the reasoning in [35]. Following the commonly adopted linear-exponential-normal (LEN) model [30] in PA theory, we assume that $D_{1}$ is linear in $e_{1}: D_{1}=e_{1}+\mu+\varepsilon_{1}$, where $\mu$ is the firm's baseline demand without sales effort and $\varepsilon_{1}$ is the random effect with normal distribution $\mathcal{N}\left(0, \sigma^{2}\right)$ denoting uncertain market conditions, and parametric values $(\mu$ and $\sigma)$ are such that the probability of negative demand is negligible. The service provider incurs quadratic cost due to sales effort: $C_{1}\left(e_{1}\right)=\frac{1}{2} k_{1} e_{1}^{2}$. We assume that the supply chain can deliver all the generated demand, which can be justified when the brand owner has other retail channels with ample supply or when the selling season includes a pre-sale period when customers place orders in advance, as is popular in e-business.

The quality-control effort level, denoted as $e_{2}$, directly influences the quality level of products and, thus, customers' post-purchase perceived quality. So without loss of generality, let $e_{2}$ represent the value of perceived quality. Now based on the disconfirmation model from Anderson and Sullivan [2], we can formulate the customer satisfaction $S$ as $S=f_{1}\left(e_{2}\right)+f_{2}\left(e_{2}-e_{1}\right)$, where $f_{1}$ describes the impact of perceived quality on CS, while $f_{2}$ characterizes the effect of disconfirmation and both are increasing functions in the model. To keep our model simple and tractable, we assume $f_{i}(i=1,2)$ are linear functions and rewrite $S=e_{2}+\theta\left(e_{2}-e_{1}\right)$ without loss of generality, where $0<\theta<1$ is called the "disconfirmation factor" (discussions on the model extension with nonlinear $f_{i}$ are given in the appendices). This formulation is similar to those in [21,35], and the assumption on $\theta$ is reasonable due to its consistency with empirical findings in [2], where the influence of disconfirmation on CS is not as much as that of perceived quality. Let $D_{2}$ denote the firm's future demand and $D_{2}=S+\mu+\varepsilon_{2}$, where $\mu$ is still the baseline demand and $\varepsilon_{2} \sim \mathcal{N}\left(0, \sigma^{2}\right)$ is the random effect, similar to the structure of $D_{1}$. $D_{2}$ reflects the long-term effect of CS on firm profitability, which is adopted in the literature and validated by empirical studies. The corresponding cost due to quality-control effort $C_{2}\left(e_{2}\right)=\frac{1}{2} k_{2} e_{2}^{2}$ is incurred. Notice that without loss of generality, the unit production cost is normalized to 0. Since we consider a single-period model and the transfer payment is delivered at the end of the period before $D_{2}$ is realized, the firm need to employ other performance measures contingent on $e_{2}$ to motivate the agent to exert the desired level of effort. As mentioned before, we assume that the firm can use the measured CS value as a proxy, which can be extracted from analyzing CS-related data collected from surveys or online ratings. We name this measurement CS index (CSI) and denote it as $S_{m}$ (measured CS), and assume $S_{m}=S+\varepsilon_{m}$ with $\varepsilon_{m} \sim \mathcal{N}\left(0, \sigma_{m}^{2}\right)$ where $\sigma_{m}$ is the CS measurement error. $\varepsilon_{m}$ is assumed to be independent of $\varepsilon_{2}$.

Following assumptions commonly adopted in the literature on compensation design, we assume that the brand owner is risk neutral and that agents are risk averse with utility function $U(x)=1-\exp (-\rho x)$, where $\rho$ is 
the coefficient of absolute risk aversion. Note that for any normally distributed random variable $X \sim \mathcal{N}\left(\mu, \sigma^{2}\right)$, $\mathbb{E}[U(X)]=1-\exp \left(-\rho \mu+\frac{1}{2} \rho^{2} \sigma^{2}\right)=1-\exp \left(-\rho\left(\mu-\frac{1}{2} \rho \sigma^{2}\right)\right)$, where the term $\mu-\frac{1}{2} \rho \sigma^{2}$ is the certainty equivalent $(\mathrm{CE})$ and maximizing the agents' expected utility is equivalent to maximizing the corresponding $\mathrm{CE}$. To facilitate further analysis, let $k=k_{1}, m=k_{2} / k_{1}, n=\sigma_{m} / \sigma, \bar{m}=\frac{(\theta+1)^{2}\left(k \rho \sigma^{2}+1\right)}{(1-\theta) \theta k \rho \sigma^{2}}, \underline{m}=\frac{(\theta+1)^{2}\left(\theta+\theta k \rho \sigma^{2}-1\right)}{(1-\theta) k \rho \sigma^{2}\left(\theta^{2}+n^{2}\right)}$. Thus $m$ is the ratio of the marginal cost of quality-control effort and sales effort and can be viewed as the relative marginal cost of quality-control activity, whereas $n$ denotes the relative CSI measure error compared with demand uncertainty. We will show in the lemma below that $\underline{m}<\bar{m}$, and we further assume $n^{2} \geq 1 / 2$ and $\underline{m}^{+} \leq m \leq \bar{m}$, where $\underline{m}^{+}=\max \{\underline{m}, 0\}$. The former assumption states that $\sigma_{m}$ cannot be very small, which is reasonable since the associated cost of increasing measure accuracy can be very high when $\sigma_{m}$ is relatively small, as described by Kung and Chen [36]. The latter assumption offers upper and lower bounds for the ratio of marginal costs of the two effort levels, which will facilitate our derivation below. We can further derive related properties on the bounds, as the lemma below shows.

Lemma 2.1. (i) $\bar{m}>\underline{m}$;

(ii) $\bar{m}$ is quasi-convex in $\theta$ and reaches its minimum $\frac{8\left(k \rho \sigma^{2}+1\right)}{k \rho \sigma^{2}}$ when $\theta=1 / 3$;

(iii) $\underline{m}^{+}$is increasing in $\theta$, and for any $m \leq \frac{8\left(k \rho \sigma^{2}+1\right)}{k \rho \sigma^{2}}$, there exists a $\bar{\theta}(m)$ such that $m$ is feasible $\left(\underline{m}^{+} \leq m \leq \bar{m}\right)$ when $\theta \in[0, \bar{\theta}(m)]$.

Given that assuming $m \leq \frac{8\left(\rho \sigma^{2}+1\right)}{\rho \sigma^{2}}$ brings more analytical convenience to our model, in the rest of the paper, we will adopt this assumption. Note that it is not strict since in general, the marginal cost of quality-control effort will not be much larger than that of sales effort.

Before delving into the detailed incentive design problem, we conclude this section by analyzing the joint marketing-operation decision problem under the centralized case as the analytical benchmark, which is formulated as

$$
\max _{e_{1}, e_{2}} \pi=\mathbb{E}\left[D_{1}+D_{2}-C_{1}\left(e_{1}\right)-C_{2}\left(e_{2}\right)\right]=e_{1}+e_{2}+\theta\left(e_{2}-e_{1}\right)-\frac{1}{2} k_{1} e_{1}^{2}-\frac{1}{2} k_{2} e_{2}^{2} .
$$

Note that without loss of generality, we can assume $\mu=0$ since the specific value of baseline demand will not affect the optimal effort levels, and we keep this assumption in the following sections. The optimal effort levels are

$$
e_{1}^{C}=\frac{1-\theta}{k_{1}}, e_{2}^{C}=\frac{1+\theta}{k_{2}},
$$

by solving the first-order conditions directly due to the profit function's joint concavity in $e_{i}(i=1,2)$, where the superscript $C$ denotes the "centralized" case. The major notations introduced in this section, which are also used throughout the paper, are summarized in Table 1.

\section{INCENTIVE DESIGN UNDER THE MULTI-TASK SCENARIO}

In this section, we consider the incentive design problem under the multi-task scenario. The sequence of events is as follows. (1) The brand owner offers a transfer payment plan contingent on specific performance measures to the agent. (2) The agent decides whether to accept it, if he rejects it, he gets his reservation utility. (3) If the agent accepts it, he exerts both sales and quality-control efforts (notice that the two activities may not be conducted simultaneously and quality-control effort is often exerted before the selling season since it takes considerable lead time). (4) Current demand materializes and CSI is measured by the brand owner. (5) The brand owner pays to the agent accordingly. In describing the transfer payment from the principal to agent, with a little misuse of the terminology, we will not distinguish between compensation and contracts, although the former is commonly used for intra-firm payment to employees while the latter are common in inter-firm scenarios. We assume that payment is composed of a fixed salary and variable commission and has a linear form: 
TABLE 1. Notations for model parameters and variables.

\begin{tabular}{ll}
\hline \hline$e_{1}$ & sales effort level \\
$e_{2}$ & Quality-control effort level \\
$\mu$ & Baseline market demand without exerting efforts \\
$D_{1}\left(D_{2}\right)$ & Market demand in the current (future) period \\
$\varepsilon_{1}\left(\varepsilon_{2}\right)$ & Random effects in market demand \\
$k_{1}\left(k_{2}\right)$ & Coefficients in cost functions for sales (quality-control) activities \\
$S$ & Customer satisfaction value \\
$f_{1}$ & The effect of perceived quality of product on customer satisfaction \\
$f_{2}$ & The effect of disconfirmation on customer satisfaction \\
$\theta$ & Disconfirmation factor \\
$S_{m}$ & Measured customer satisfaction or customer satisfaction index (CSI) \\
$\varepsilon_{m}$ & Customer satisfaction measurement error \\
$m$ & $k_{2} / k_{1}$, the relative marginal cost of quality-control effort \\
$n$ & $\varepsilon_{m} / \varepsilon$, the relative CSI measure error \\
$\rho$ & Coefficient of absolute risk aversion \\
\hline
\end{tabular}

$T=\alpha+\beta_{1} D_{1}+\beta_{2} S_{m}$. Thus the performance measures are current demand (sales) and CSI, which depend on the specific value of effort levels. $\alpha$ is the fixed payment, $\beta_{1}$ and $\beta_{2}$ are commission rates for different measures.

We can calculate the agent's CE as

$$
\alpha+\beta_{1} e_{1}+\beta_{2}\left(e_{2}+\theta\left(e_{2}-e_{1}\right)\right)-\frac{1}{2} \rho\left(\beta_{1}^{2} \sigma^{2}+\beta_{2}^{2} \sigma_{m}^{2}\right)-\frac{1}{2} k_{1} e_{1}^{2}-\frac{1}{2} k_{2} e_{2}^{2} .
$$

Following the standard PA framework, the incentive design problem can be formulated as

$$
\begin{aligned}
\max _{\alpha, \beta_{1}, \beta_{2}} & =e_{1}^{*}+e_{2}^{*}+\theta\left(e_{2}^{*}-e_{1}^{*}\right)-\left(\alpha+\beta_{1} e_{1}^{*}+\beta_{2}\left(e_{2}^{*}+\theta\left(e_{2}^{*}-e_{1}^{*}\right)\right)\right) \\
\text { s.t. } \quad & e_{i}^{*}=\underset{e_{i}}{\arg \max } \alpha+\beta_{1} e_{1}+\beta_{2}\left(e_{2}+\theta\left(e_{2}-e_{1}\right)\right)-\frac{1}{2} \rho\left(\beta_{1}^{2} \sigma^{2}+\beta_{2}^{2} \sigma_{m}^{2}\right)-\frac{1}{2} k_{1} e_{1}^{2}-\frac{1}{2} k_{2} e_{2}^{2}, i=1,2 \\
& \alpha+\beta_{1} e_{1}+\beta_{2}\left(e_{2}+\theta\left(e_{2}-e_{1}\right)\right)-\frac{1}{2} \rho\left(\beta_{1}^{2} \sigma^{2}+\beta_{2}^{2} \sigma_{m}^{2}\right)-\frac{1}{2} k_{1} e_{1}^{2}-\frac{1}{2} k_{2} e_{2}^{2} \geq 0
\end{aligned}
$$

where the firm's profit $\pi$ is the total revenue earned in the current and future periods when the effort levels are exerted in the best interest of the agent less the total payment to the agent. The constraint (2) is the incentive compatibility (IC) condition corresponding to the agent's optimal effort levels given contract parameters, and constraint (3) is the individual rationality (IR) condition ensuring the agent's participation. Without loss of generality, we let the agent's reservation utility, i.e., the right-hand side of inequality (3) be 0 , which is commonly adopted in the literature. To solve the problem, following the standard procedure in solving the PA model, we derive $e_{i}^{*}$ from the first-order conditions of the IC constraint since the agent's income is jointly concave in $\left\{e_{1}, e_{2}\right\}$. We then let the IR constraint be binding and substitute all the results back to the principal's profit function, which can be represented as a function of $\left(\beta_{1}, \beta_{2}\right)$. Then, we can derive the optimal contract parameters. The following theorem shows the analytical results, and note that we attach superscript MT to all optimal solutions in this section, which stands for "multi-task".

Theorem 3.1. The brand owner's profit is jointly concave in $\beta_{1}$ and $\beta_{2}$; the optimal commission rates, effort levels and the brand owner's profit are shown in Table 2.

We further investigate how the optimal solutions and profit change with varying model parameters, and the following proposition shows the result. Note that since we assume that there exists a constraint between $m$ and $\theta\left(\underline{m}^{+} \leq m \leq \bar{m}\right)$, when we talk about any quantity "increasing/decreasing in $m($ or $\theta)$ ", we mean that the 
TABLE 2. Optimal commission rates, effort levels and profit for the multi-task scenario.

\begin{tabular}{ll}
\hline \hline$\beta_{1}^{\mathrm{MT}}$ & $\frac{(\theta+1)^{2}+(1-\theta) k m n^{2} \rho \sigma^{2}}{(\theta+1)^{2}+k^{2} m n^{2} \rho^{2} \sigma^{4}+k \rho \sigma^{2}\left((\theta+1)^{2}+m\left(\theta^{2}+n^{2}\right)\right)}$ \\
$\beta_{2}^{\mathrm{MT}}$ & $\frac{(\theta+1)^{2}+k \rho \sigma^{2}(\theta(\theta+(\theta-1) m+2)+1)}{(\theta+1)^{2}+k^{2} m n^{2} \rho^{2} \sigma^{4}+k \rho \sigma^{2}\left((\theta+1)^{2}+m\left(\theta^{2}+n^{2}\right)\right)}$ \\
$e_{1}^{\mathrm{MT}}$ & $-\frac{(\theta-1)(\theta+1)^{2}+k \rho \sigma^{2}\left(\theta(\theta+1)^{2}+(\theta-1) m\left(\theta^{2}+n^{2}\right)\right)}{k\left((\theta+1)^{2}+k^{2} m n^{2} \rho^{2} \sigma^{4}+k \rho \sigma^{2}\left((\theta+1)^{2}+m\left(\theta^{2}+n^{2}\right)\right)\right)}$ \\
$e_{2}^{\mathrm{MT}}$ & $\frac{(\theta+1)\left((\theta+1)^{2}+k \rho \sigma^{2}(\theta(\theta+(\theta-1) m+2)+1)\right)}{k m\left((\theta+1)^{2}+k^{2} m n^{2} \rho^{2} \sigma^{4}+k \rho \sigma^{2}\left((\theta+1)^{2}+m\left(\theta^{2}+n^{2}\right)\right)\right)}$ \\
$\pi^{\mathrm{MT}}$ & $\frac{(\theta+1)^{4}\left(k \rho \sigma^{2}+1\right)+(\theta-1)^{2} k m^{2} \rho \sigma^{2}\left(\theta^{2}+n^{2}\right)+(\theta-1)(\theta+1)^{2} m\left(\theta+2 \theta k \rho \sigma^{2}-1\right)}{2 k m\left((\theta+1)^{2}+k^{2} m n^{2} \rho^{2} \sigma^{4}+k \rho \sigma^{2}\left((\theta+1)^{2}+m\left(\theta^{2}+n^{2}\right)\right)\right)}$ \\
\hline
\end{tabular}

quantity has this property in the feasible region of $m($ or $\theta$ ), given the predetermined value of $\theta$ (or $m$ ) (with the assumption $m \leq \frac{8\left(\rho \sigma^{2}+1\right)}{\rho \sigma^{2}}$ we can ensure such regions are convex and closed due to the monotonicity of $\left.\bar{\theta}(m)\right)$.

Proposition 3.2. (i) $\beta_{i}^{\mathrm{MT}} \geq 0, e_{i}^{C} \geq e_{i}^{\mathrm{MT}} \geq 0(i=1,2)$.

(ii) $\beta_{i}^{\mathrm{MT}}(i=1,2)$ is decreasing in $n, m, k, \rho, \sigma ; \beta_{1}^{\mathrm{MT}}$ is decreasing in $\theta ; \beta_{2}^{\mathrm{MT}}$ is quasi-convex in $\theta ; \beta_{2}^{\mathrm{MT}}$ is increasing in $\theta$ when $\theta \geq 1 / 2$ or $m<\frac{2 n^{2}-1}{k n^{2} \rho \sigma^{2}}+2$.

(iii) $\pi^{\mathrm{MT}}$ is decreasing in $n, m$.

(iv) The benefit of increasing CSI measure accuracy is more significant when the marginal cost of sales effort is relatively larger than the marginal cost of quality-control effort and when the disconfirmation factor $\theta$ is larger given $\theta \geq 1 / 2$.

Part (ii) of the proposition shows that both the optimal commission rates decrease in the relative marginal cost of quality-control effort and relative CSI measure error. This is intuitive for $\beta_{2}^{\mathrm{MT}}$ and slightly interesting for $\beta_{1}^{\mathrm{MT}}$ since by expectation, more incentives should be placed on current demand $D_{1}$. In fact, however, we find that $e_{1}^{\mathrm{MT}}$ is increasing in $m$ and $n$ :

$$
\begin{aligned}
\frac{\partial e_{1}^{\mathrm{MT}}}{\partial m} & =\frac{\theta(\theta+1)^{2} k \rho^{2} \sigma^{4}\left(\theta+n^{2}\left(k \rho \sigma^{2}+1\right)\right)}{\left((\theta+1)^{2}+k^{2} m n^{2} \rho^{2} \sigma^{4}+k \rho \sigma^{2}\left((\theta+1)^{2}+m\left(\theta^{2}+n^{2}\right)\right)\right)^{2}}>0, \\
\frac{\partial e_{1}^{\mathrm{MT}}}{\partial n} & =\frac{2 \theta k m n \rho^{2} \sigma^{4}}{(\theta+1)^{2}+k^{2} m n^{2} \rho^{2} \sigma^{4}+k \rho \sigma^{2}\left((\theta+1)^{2}+m\left(\theta^{2}+n^{2}\right)\right)} \beta_{2}^{\mathrm{MT}}>0 .
\end{aligned}
$$

Therefore, when model parameters are such that exerting sales effort is more cost-effective, the commission rates will be well balanced to facilitate this trend. $\beta_{1}^{\mathrm{MT}}$ does not need to increase with $m$ and $n$ because otherwise, $e_{1}^{\mathrm{MT}}$ may be too large to reach the appropriate level and the firm will also incur a greater risk premium. We also find $\frac{\partial^{2} \pi}{\partial \beta_{1} \partial \beta_{2}}=\theta / k_{1}>0$, so the commission rates have a complementary effect on the profit; thus, it is reasonable that they move in the same direction when certain parameters change.

We also find that the disconfirmation factor $\theta$ plays a subtle role in determining the optimal commission rates and profit. The result demonstrates consistency with the argument in [21], which emphasizes the critical role played by the spoiling effect. It is natural to expect $\beta_{1}^{\mathrm{MT}}$ to decrease in $\theta$ since a higher $\theta$ motivates the firm to focus more on disconfirmation and put less emphasis on sales effort. The case is more intricate for $\beta_{2}^{\mathrm{MT}}$. Now, when the marginal cost ratio $m$ is large, with gradually increasing $\theta, \beta_{2}^{\mathrm{MT}}$ will first decrease. Our explanation is that higher disconfirmation can be enabled with either lower $e_{1}^{\mathrm{MT}}$ or higher $e_{2}^{\mathrm{MT}}$, but the change in $\beta_{1}^{\mathrm{MT}}$ with $\theta$ have already lowered $e_{1}^{\mathrm{MT}}$. From $e_{2}^{*}=\frac{\beta_{2}(\theta+1)}{k_{2}}$, we see that given $\beta_{2}$, the optimal quality-control effort level is increasing in $\theta$. So given the high marginal cost of quality-control effort, decreasing $\beta_{2}^{\mathrm{MT}}$ can balance the 

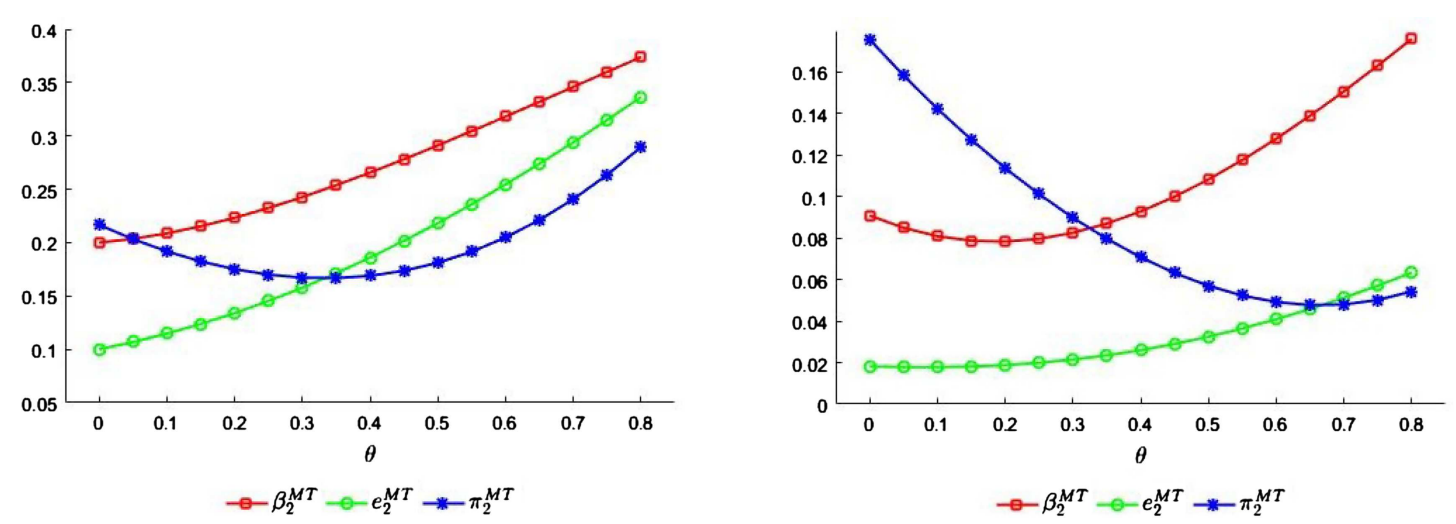

Figure 1. $\beta_{2}^{\mathrm{MT}}, e_{2}^{\mathrm{MT}}, \pi^{\mathrm{MT}}$ varying with $\theta$ when $m=2$ (left) and $m=5$ (right) with fixed parametric values $n=1, k=2, \rho=0.5, \sigma=2$.

benefit of utilizing disconfirmation and the considerable cost and the risk premium paid. However, when $\theta$ is large enough, disconfirmation is more critical in determining CS, so $\beta_{2}^{\mathrm{MT}}$ increases in $\theta$. Figure 1 numerically shows how $\beta_{2}^{\mathrm{MT}}, e_{2}^{\mathrm{MT}}$ and $\pi^{\mathrm{MT}}$ vary with $\theta$ for different $m$, given other model parameters. For the left panel, where $m=2$, we find both $\beta_{2}^{\mathrm{MT}}$ and $e_{2}^{\mathrm{MT}}$ increasing in $\theta$, while they behave like quasi-convex functions in $\theta$ when $m=5$ for the right panel and the values of these quantities are smaller than their counterparts when $m=2$. We also observe that the profit decreases in $\theta$ first then increases, showing a quasi-convex figure. This finding can be attributed to the fact that when $\theta$ is sufficiently small or large, the dominant determinant of profit is either sales or quality-control effort and the firm can fully utilize the corresponding effort to reach high profit. Whereas more consideration is made to balance two effort levels when $\theta$ has an intermediate value.

We have shown that increasing the CSI measurement accuracy brings benefit since the firm will pay less risk premium to the agent, and this benefit can vary with different model parameters. It is natural to expect that the benefit become more profound when marginal cost of quality-control effort is relatively small, as is shown by part (iv) of the proposition. Because in that case, the commission rate associated with CSI in compensation is higher and the risk premium paid grows with this rate. While the benefit changes with $\theta$ in a rate proportional to $\frac{\partial \beta_{2}^{\mathrm{MT}}}{\partial \theta}$ (in fact, we can derive $-\frac{\partial^{2} \pi^{\mathrm{MT}}}{\partial n \partial \theta}=2 n \rho \sigma^{2} \beta_{2}^{\mathrm{MT}} \frac{\partial \beta_{2}^{\mathrm{MT}}}{\partial \theta}$ ). So from our analysis of $\frac{\partial \beta_{2}^{\mathrm{MT}}}{\partial \theta}$ above, we see that only when $\theta$ is relatively large or $m$ is relatively small will this benefit be more significant with an increasing $\theta$. In practice, increasing CSI measure accuracy may incur a considerable implementation cost, so for brand owners, it is advisable to consider system factors such as $\theta$ and $m$ before starting a measure error reduction initiative to balance the associated benefits and costs.

It is also interesting to investigate when the firm will place a greater incentive on either of the two performance measures, i.e., the relationship between commission rates. We find that

$$
\beta_{1}^{\mathrm{MT}}-\beta_{2}^{\mathrm{MT}}=-\frac{k \rho \sigma^{2}\left((\theta+1)^{2}+(\theta-1) m\left(\theta+n^{2}\right)\right)}{(\theta+1)^{2}+k^{2} m n^{2} \rho^{2} \sigma^{4}+k \rho \sigma^{2}\left((\theta+1)^{2}+m\left(\theta^{2}+n^{2}\right)\right)},
$$

which can be shown increasing in $m$ and $n$. In fact, denoting this difference as $\Delta \beta^{\mathrm{MT}}$, we have

$$
\begin{aligned}
& \frac{\partial \Delta \beta^{\mathrm{MT}}}{\partial m}=\frac{(\theta+1)^{2} k \rho \sigma^{2}\left(-\theta+k \rho \sigma^{2}+1\right)\left(\theta+n^{2}\left(k \rho \sigma^{2}+1\right)\right)}{\left((\theta+1)^{2}+k^{2} m n^{2} \rho^{2} \sigma^{4}+k \rho \sigma^{2}\left((\theta+1)^{2}+m\left(\theta^{2}+n^{2}\right)\right)\right)^{2}}>0, \\
& \frac{\partial \Delta \beta^{\mathrm{MT}}}{\partial n}=\frac{2 k m n \rho \sigma^{2}\left(-\theta+k \rho \sigma^{2}+1\right)}{(\theta+1)^{2}+k^{2} m n^{2} \rho^{2} \sigma^{4}+k \rho \sigma^{2}\left((\theta+1)^{2}+m\left(\theta^{2}+n^{2}\right)\right)} \beta_{2}^{\mathrm{MT}}>0 .
\end{aligned}
$$

The following corollary summarizes the parameter settings under which either effort will receive more emphasis. 
Corollary 3.3. (i) When $m$ increases, $\Delta \beta^{\mathrm{MT}}$ changes from negative to positive and crosses 0 at a threshold $m_{t}=\frac{(\theta+1)^{2}}{(1-\theta)\left(\theta+n^{2}\right)}$

(ii) $m_{t}$ is increasing in $\theta$ and decreasing in $n$.

From the corollary we can find $\beta_{1}^{\mathrm{MT}}$ is greater than $\beta_{2}^{\mathrm{MT}}$ when $m$ and $n$ are small and $\theta$ is high. Thus when the marginal cost of quality-control effort is relatively small, when the CSI measure is relatively precise, and when disconfirmation is relatively critical in determining CS, more attention will be paid to promoting quality-control effort, which is quite intuitive.

\section{INCENTIVE DESIGN UNDER THE MULTI-AGENT SCENARIO}

This section will study the incentive design problem under the multi-agent scenario where the firm contracts with MSP and OSP separately. We first consider a basic model where the MSP is compensated on $D_{1}$ while the OSP receives payment on CSI; then we investigate how results may change when the MSP is provided an extra payment contingent on CSI.

\subsection{Basic model}

We use subscript 1(2) to denote certain quantities related to the MSP (OSP). Again assume that the brand owner offers linear contracts to agents: $T_{1}=\alpha_{1}+\beta_{1} D_{1}, T_{2}=\alpha_{2}+\beta_{2} S_{m}$, and the sequence of events are almost the same as those we described in Section 3, except for Step (3), where the sales and quality-control efforts are carried out separately. Now the agents' respective certainty equivalents are

$$
C E_{1}=\alpha_{1}+\beta_{1} e_{1}-\frac{1}{2} \rho \beta_{1}^{2} \sigma^{2}-\frac{1}{2} k_{1} e_{1}^{2}, \quad C E_{2}=\alpha_{2}+\beta_{2}\left(e_{2}+\theta\left(e_{2}-e_{1}\right)\right)-\frac{1}{2} \rho \beta_{2}^{2} \sigma_{m}^{2}-\frac{1}{2} k_{2} e_{2}^{2} .
$$

While the brand owner faces a multiple-agent moral hazard problem:

$$
\begin{aligned}
\max _{\alpha_{i}, \beta_{i}} & \pi=e_{1}^{*}+e_{2}^{*}+\theta\left(e_{2}^{*}-e_{1}^{*}\right)-\left(\alpha_{1}+\beta_{1} e_{1}^{*}+\alpha_{2}+\beta_{2}\left(e_{2}^{*}+\theta\left(e_{2}^{*}-e_{1}^{*}\right)\right)\right) \\
\text { s.t. } & e_{1}^{*}=\underset{e_{1}}{\arg \max } \alpha_{1}+\beta_{1} e_{1}-\frac{1}{2} \rho \beta_{1}^{2} \sigma^{2}-\frac{1}{2} k_{1} e_{1}^{2} \\
& \alpha_{1}+\beta_{1} e_{1}-\frac{1}{2} \rho \beta_{1}^{2} \sigma^{2}-\frac{1}{2} k_{1} e_{1}^{2} \geq 0 \\
& e_{2}^{*}=\underset{e_{2}}{\arg \max } \alpha_{2}+\beta_{2}\left(e_{2}+\theta\left(e_{2}-e_{1}\right)\right)-\frac{1}{2} \rho \beta_{2}^{2} \sigma_{m}^{2}-\frac{1}{2} k_{2} e_{2}^{2}, \\
& \alpha_{2}+\beta_{2}\left(e_{2}+\theta\left(e_{2}-e_{1}\right)\right)-\frac{1}{2} \rho \beta_{2}^{2} \sigma_{m}^{2}-\frac{1}{2} k_{2} e_{2}^{2} \geq 0 .
\end{aligned}
$$

We can solve this problem with a similar technique to that introduced in the previous section, and the results are as follows.

Theorem 4.1. The brand owner's profit is jointly concave in $\beta_{1}$ and $\beta_{2}$; the optimal commission rates, effort levels and the brand owner's profit are as follows:

$$
\begin{gathered}
\beta_{1}^{\mathrm{MA}}=\frac{1-\theta}{k \rho \sigma^{2}+1}, \beta_{2}^{\mathrm{MA}}=\frac{(\theta+1)^{2}}{(\theta+1)^{2}+k m n^{2} \rho \sigma^{2}}, e_{1}^{\mathrm{MA}}=\frac{1-\theta}{k^{2} \rho \sigma^{2}+k}, \\
e_{2}^{\mathrm{MA}}=\frac{(\theta+1)^{3}}{k m\left((\theta+1)^{2}+k m n^{2} \rho \sigma^{2}\right)}, \pi^{\mathrm{MA}}=\frac{1}{2 k}\left(\frac{(\theta-1)^{2}}{k \rho \sigma^{2}+1}+\frac{(\theta+1)^{4}}{m\left((\theta+1)^{2}+k m n^{2} \rho \sigma^{2}\right)}\right) .
\end{gathered}
$$

The superscript MA for all optimal quantities in this subsection stands for "multi-agent". Regarding how the optimal solutions are affected by varying model parameters, we have the following proposition. 
TABLE 3. Sensitivity analysis of optimal solutions for the basic multi-agent model.

\begin{tabular}{lllllll}
\hline \hline & $m$ & $n$ & $\theta$ & $k$ & $\rho$ & $\sigma$ \\
\hline$\beta_{1}^{\mathrm{MA}}$ & - & - & $\downarrow$ & $\downarrow$ & $\downarrow$ & $\downarrow$ \\
$\beta_{2}^{\mathrm{MA}}$ & $\downarrow$ & $\downarrow$ & $\uparrow$ & $\downarrow$ & $\downarrow$ & $\downarrow$ \\
$e_{1}^{\mathrm{MA}}$ & - & - & $\downarrow$ & $\downarrow$ & $\downarrow$ & $\downarrow$ \\
$e_{2}^{\mathrm{MA}}$ & $\downarrow$ & $\downarrow$ & $\uparrow$ & $\downarrow$ & $\downarrow$ & $\downarrow$ \\
$\pi^{\mathrm{MA}}$ & $\downarrow$ & $\downarrow$ & $\sim$ & $\downarrow$ & $\downarrow$ & $\downarrow$ \\
\hline
\end{tabular}

Proposition 4.2. (i) The effects of varying parameters on optimal decisions and profit are shown in Table 3, where " $\uparrow, \downarrow,-, \sim$ " stand for increasing, decreasing, independent, and unclear, respectively; $\pi^{\mathrm{MA}}$ is convex in $\theta$; when $m$ and $n$ are small enough, $\pi^{\mathrm{MA}}$ is increasing in $\theta$.

(ii) The benefit of increasing CSI measure accuracy is more significant when the marginal cost of sales effort is relatively larger than the marginal cost of quality-control effort and when the disconfirmation factor is larger.

Compared with the multi-task model, this model emphasizes the separation of sales and quality-control efforts between different agents; hence, the conclusions from the proposition are straightforward. First note that sales effort level is independent of the relative measure accuracy of CSI and the relative marginal cost of qualitycontrol effort. From $\frac{\partial^{2} \pi\left(\beta_{1}, \beta_{2}\right)}{\partial \beta_{1} \partial \beta_{2}}=0$, we can see there is less interaction between the commission rates and they influence the optimal profit separately. Now, since how the two effort levels change with $\theta$ is clear, the evolution of $\pi^{\mathrm{MA}}$ in $\theta$ is driven mainly by the relatively dominant relationship between $e_{1}^{\mathrm{MA}}$ and $e_{2}^{\mathrm{MA}}$ with varying $\theta$ due to their monotonicity. For moderate values of $m$ and $n$, when $\theta$ is sufficiently small or large, the major determinant of profit is either sales effort or quality-control effort, and the firm can take full advantage of the dominant effort to keep profit high. When $\theta$ is at an intermediate level, no clear dominance is observed, and the profit is relatively low. However, when $m$ and $n$ are sufficiently small, the effect of quality-control effort will always be dominant, causing $\pi^{\mathrm{MA}}$ to increase in $\theta$. Part (ii) of the proposition finds that the impact of increasing measure accuracy on profit will be more significant when carrying out quality-control activity is more cost effective and when disconfirmation is more important in CS determination since only the OSP is compensated on CSI, and both cases will increase the associated commission rate $\beta_{2}^{\mathrm{MA}}$.

\subsection{Incentivizing MSP with an extra term on CSI}

The classical literature on the moral hazard problem has shown that when more information related to the agent's unobserved action is introduced into the incentive scheme, the principal will benefit from this information gain. Since CS is jointly determined by sales and quality-control efforts, in this subsection, we proceed to explore how including an extra term on CSI in the MSP's compensation package will change the analytical results.

Now the MSP is offered a new contract $T_{1}=\alpha_{1}+\beta_{1} D_{1}+\gamma S_{m}$ where $\gamma$ is the extra commission rate on CSI, and the corresponding $\mathrm{CE}$ equals

$$
\alpha_{1}+\beta_{1} e_{1}+\gamma\left(e_{2}+\theta\left(e_{2}-e_{1}\right)\right)-\frac{1}{2} \rho \beta_{1}^{2} \sigma^{2}-\frac{1}{2} \rho \gamma^{2} \sigma_{m}^{2}-\frac{1}{2} k_{1} e_{1}^{2} .
$$


TABLE 4. Sensitivity analysis of optimal solutions for the multi-agent model with a CSI incentive for the MSP.

\begin{tabular}{lcccccc}
\hline \hline & $m$ & $n$ & $\theta$ & $k$ & $\rho$ & $\sigma$ \\
\hline$\beta_{1}^{\text {MAS }}$ & - & $\uparrow$ & $\downarrow$ & $\downarrow$ & $\downarrow$ & $\downarrow$ \\
$\gamma^{\text {MAS }}$ & - & $\uparrow$ & $\sim$ & $\uparrow$ & $\uparrow$ & $\uparrow$ \\
$\beta_{2}^{\text {MAS }}$ & $\downarrow$ & $\downarrow$ & $\uparrow$ & $\downarrow$ & $\downarrow$ & $\downarrow$ \\
$e_{1}^{\text {MAS }}$ & - & $\downarrow$ & $\downarrow$ & $\downarrow$ & $\downarrow$ & $\downarrow$ \\
$e_{2}^{\text {MAS }}$ & $\downarrow$ & $\downarrow$ & $\uparrow$ & $\downarrow$ & $\downarrow$ & $\downarrow$ \\
$\pi^{\text {MAS }}$ & $\downarrow$ & $\downarrow$ & $\sim$ & $\downarrow$ & $\downarrow$ & $\downarrow$ \\
\hline
\end{tabular}

The incentive problem can be formulated as

$$
\begin{array}{ll}
\max _{\alpha_{i}, \beta_{i}, \gamma} & \pi=e_{1}^{*}+e_{2}^{*}+\theta\left(e_{2}^{*}-e_{1}^{*}\right)-\left(\alpha_{1}+\beta_{1} e_{1}+\alpha_{2}+\left(\beta_{2}+\gamma\right)\left(e_{2}^{*}+\theta\left(e_{2}^{*}-e_{1}^{*}\right)\right)\right) \\
\text { s.t. } & e_{1}^{*}=\underset{e_{1}}{\arg \max } \alpha_{1}+\beta_{1} e_{1}+\gamma\left(e_{2}+\theta\left(e_{2}-e_{1}\right)\right)-\frac{1}{2} \rho \beta_{1}^{2} \sigma^{2}-\frac{1}{2} \rho \gamma^{2} \sigma_{m}^{2}-\frac{1}{2} k_{1} e_{1}^{2}, \\
& \alpha_{1}+\beta_{1} e_{1}+\gamma\left(e_{2}+\theta\left(e_{2}-e_{1}\right)\right)-\frac{1}{2} \rho \beta_{1}^{2} \sigma^{2}-\frac{1}{2} \rho \gamma^{2} \sigma_{m}^{2}-\frac{1}{2} k_{1} e_{1}^{2} \geq 0 \\
& e_{2}^{*}=\underset{e_{2}}{\arg \max } \alpha_{2}+\beta_{2}\left(e_{2}+\theta\left(e_{2}-e_{1}\right)\right)-\frac{1}{2} \rho \beta_{2}^{2} \sigma_{m}^{2}-\frac{1}{2} k_{2} e_{2}^{2} \\
& \alpha_{2}+\beta_{2}\left(e_{2}+\theta\left(e_{2}-e_{1}\right)\right)-\frac{1}{2} \rho \beta_{2}^{2} \sigma_{m}^{2}-\frac{1}{2} k_{2} e_{2}^{2} \geq 0 .
\end{array}
$$

The optimal solutions and the profit are shown in Theorem 4.3 below. The superscript $M A S$ for all optimal quantities in this subsection stands for "multi-agent with satisfaction incentive".

Theorem 4.3. The brand owner's profit is jointly concave in $\beta_{1}, \beta_{2}, \gamma$, and the optimal commission rates, effort levels and the brand owner's profit are, respectively,

$$
\begin{aligned}
& \beta_{1}^{\mathrm{MAS}}=-\frac{(\theta-1) n^{2}}{\theta^{2}+n^{2}\left(k \rho \sigma^{2}+1\right)}, \gamma^{\mathrm{MAS}}=\frac{(\theta-1) \theta}{\theta^{2}+n^{2}\left(k \rho \sigma^{2}+1\right)}, \beta_{2}^{\mathrm{MAS}}=\frac{(\theta+1)^{2}}{(\theta+1)^{2}+k m n^{2} \rho \sigma^{2}} \\
& e_{1}^{\mathrm{MAS}}=-\frac{(\theta-1)\left(\theta^{2}+n^{2}\right)}{k\left(\theta^{2}+n^{2}\left(k \rho \sigma^{2}+1\right)\right)}, e_{2}^{\mathrm{MAS}}=\frac{(\theta+1)^{3}}{k m\left((\theta+1)^{2}+k m n^{2} \rho \sigma^{2}\right)}, \\
& \pi_{P}^{\mathrm{MAS}}=\frac{1}{2 k}\left(\frac{(\theta+1)^{4}}{m\left((\theta+1)^{2}+k m n^{2} \rho \sigma^{2}\right)}+\frac{(\theta-1)^{2}\left(\theta^{2}+n^{2}\right)}{\theta^{2}+n^{2}\left(k \rho \sigma^{2}+1\right)}\right) .
\end{aligned}
$$

Regarding how the optimal solutions are affected by varying model parameters, we have the following proposition.

Proposition 4.4. (i) The effects of varying parameters on optimal decisions and profit is shown in Table 4, where " $\uparrow, \downarrow,-, \sim$ " stand for increasing, decreasing, independent, and unclear, respectively; $\gamma^{\mathrm{MAS}}$ is quasiconvex in $\theta ; \pi^{\mathrm{MAS}}$ is convex in $\theta$ and when $m$ and $n$ are small enough; $\pi^{\mathrm{MAS}}$ is increasing in $\theta$.

(ii) The benefit of increasing CSI measure accuracy is more significant when the marginal cost of sales effort is relatively larger than the marginal cost of quality-control effort and when the disconfirmation factor $\theta$ is larger given that $\theta$ is small enough (specifically when $\theta<\frac{1}{2}(\sqrt{3}-1)$ ).

Most of the sensitivity analyses for optimal solutions here are similar to those in the basic case in the last subsection, and similar explanations can be provided. The quasi-convexity of $\gamma^{\mathrm{MAS}}$ in $\theta$ has a similar 
interpretation as that of $\beta_{2}^{\mathrm{MT}}$ in the multi-task case, reflecting how commission rates are carefully manipulated by the brand owner, causing $e_{1}^{\mathrm{MAS}}$ to decrease with $\theta$ in a cost-effective way. Similar to the basic case and the multi-task case, the benefit from increasing CSI measure accuracy will be higher when marginal cost of quality-control effort is relatively smaller. But for factor $\theta$, the result derived in the basic case is observed only when $\theta$ is small, which is contrary to the result observed for the multi-task case. Therefore, again, when the brand owner sets out to reduce the CSI measure error, a better knowledge of the values of environmental factors is required to fully utilize the benefit.

\section{Comparison of the Results}

We have investigated the optimal compensation design under both the multi-task case and the multi-agent case (including two subcases where the MSP is paid and not paid on CSI), while how the firm's performance, especially the optimal profit, vary for these cases and the critical roles played by model parameters in influencing the difference remain to be answered. We will carry out a related analysis in this section.

We first consider the multi-agent case studied in Section 4 and compare the basic model with that including CSI incentive in the MSP's compensation. Several findings are shown in the following proposition.

Proposition 5.1. The brand owner will earn extra profit by incentivizing the MSP on CSI under the multiagent case; the benefit is independent of $m$, decreasing in $n$ and $k$ and unimodal in $\rho, \theta, \sigma$ and has an upper bound approximating $0.013 / k$ with a fixed $k$.

The proposition first shows the existence of extra benefit when the MSP is compensated not only on current demand bust also customer satisfaction, which is consistent with the literature on contract theory, given that more information on the MSP's effort level can be extracted. It also implies that the marginal cost of qualitycontrol effort does not affect the benefit, which is quite intuitive since the extra incentive term on CSI is offered to the MSP and does not affect the OSP's decision. We also find that when the marginal cost of sales effort is high or the CSI measure is not particularly accurate, the benefit is quite small. In fact, it can be shown that even if we relax the assumption $n^{2} \geq 1 / 2$ and allow $n$ to be any positive number, an upper bound for the benefit that equals $\frac{1}{2 k}$ still exists when $n \rightarrow 0$, so the absolute value of improvement will not be as significant as anticipated under certain parameter settings. Since including CSI in the MSP's compensation plan may incur additional transactional and implementation costs, the firm should be cautious in trading off the benefit and cost under certain environments.

Although the absolute benefit is bounded and can even be negligible for certain parameter settings, the relative benefit may be different. Since the derivation of clear analytical results regarding relative profit gain is not easy, we carry out corresponding numerical studies instead. Let $\delta \pi=\pi^{\mathrm{MAS}}-\pi^{\mathrm{MA}}$ and $\Delta \pi=\delta \pi / \pi^{\mathrm{MA}}$ denote the absolute and relative profit gains and how $\Delta \pi$ changes with parameters $\theta, m, n$ is shown in Figure 2 . It is observed that $\Delta \pi$ is unimodal in $\theta$, decreasing in $n$ and increasing in $m$ (the monotonicity in $m$ can in fact be shown analytically since $\delta \pi$ is irrelevant with $m$ while $\pi^{\mathrm{MA}}$ is decreasing in $m$ ), and for certain parameter settings, the benefit can reach as much as $20 \%$, which is a considerable improvement. So in the relative sense, compensating the MSP on CSI can be a tactic worth trying.

In the rest of this section, we focus on the comparison between the firm's decisions and profits under the multi-task and basic multi-agent case. At first glance, it is not straightforward to claim if there exists any dominant relationship between the two cases. On one hand, when both sales and quality-control activities are carried out by a single agent, there may be more latitude for coordination with an integrated decision-making process for both efforts; on the other hand, when delegating jobs to separate agents, the brand owner has more flexibility in incentive design and can somehow benefit from decoupling different efforts. Therefore, it remains unclear which outsourcing model may dominate. However, we find a quite interesting result revealed by the proposition below.

Theorem 5.2. The brand owner always earns more profit under the multi-agent case than under the multi-task case, i.e., $\pi^{\mathrm{MA}}>\pi^{\mathrm{MT}}$. 

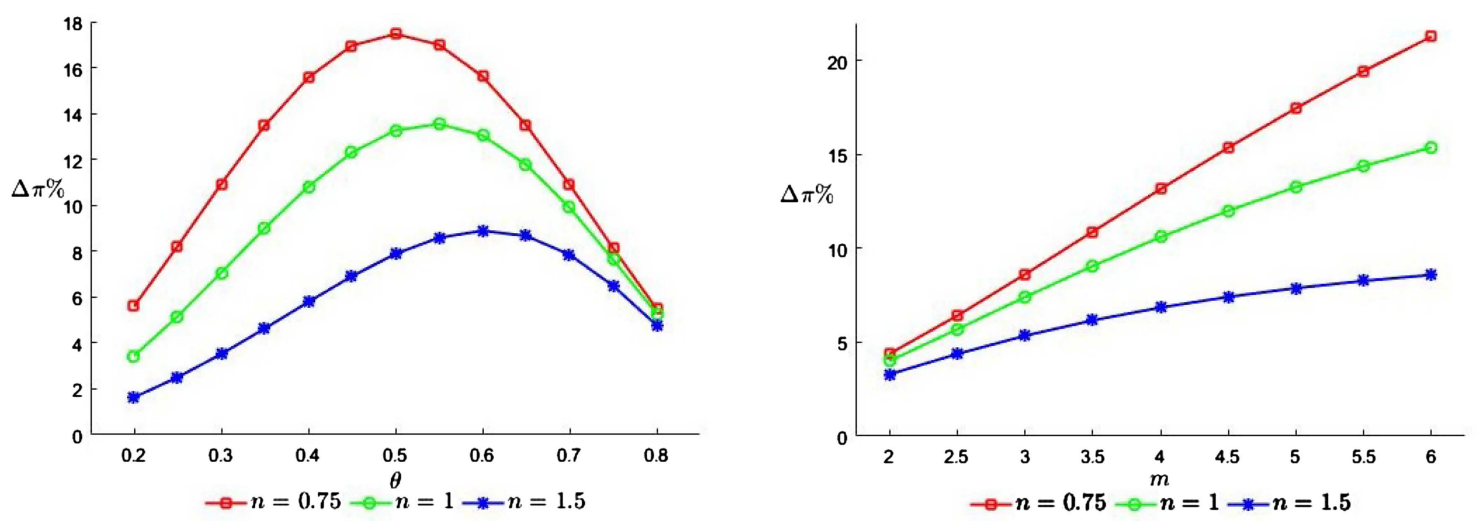

FIGURE 2. Relative profit gain varying with $\theta$ (left) and $m$ (right) with $n=0.75,1,1.5$ and base parameter values $\theta=0.5, m=5, k=2, \rho=2, \sigma=3$.

It turns out that the profit made under the multi-agent scenario will always outperform that under the multitask scenario. Our explanation for this conclusion is that in the multi-task case, the conflicting nature of the two performance measures due to the disconfirmation model (sales effort increases current demand but decreases CS) will bring a lower degree of freedom in incentivizing the single agent to exert the effort levels the brand owner desires. For example, when marginal cost of quality-control effort is lower or the CSI measure is more accurate ( $m$ or $n$ is smaller), the brand owner will adjust the commission rates to induce higher quality-control effort. Under the basic multi-agent case, the adjustment will affect only $e_{2}$. While for the multi-task case, higher $\beta_{2}$ will influence both $e_{1}$ and $e_{2}$, so the brand owner may even need to adjust $\beta_{1}$ to reset the system in a balancing state. Such interdependence in decision making under the multi-task case will make the brand owner more prudent in changing commission rates, which restricts the possibility of making more profit.

In Section 6.2.2 of [5], the authors consider a contract design problem where a principal delegates two conflicting tasks to agents. They assume that more effort exerted on one task will affect its outcome positively but affect the other task negatively, and find that when the conflict is serious enough, hiring two agents responsible for separate tasks will generate more benefit for the principal than hiring a multitasking agent. Our model setting is quite similar to this problem, so some consistency in analytical results can also be found.

To further understand the driving force of the profit difference between the two outsourcing models, we focus on the comparison of optimal commission rates and effort levels between the two cases, with results shown in the following corollary.

Corollary 5.3. $\beta_{1}^{\mathrm{MA}} \leq \beta_{1}^{\mathrm{MT}}, \beta_{2}^{\mathrm{MA}} \geq \beta_{2}^{\mathrm{MT}} ; e_{1}^{\mathrm{MA}} \geq e_{1}^{\mathrm{MT}}, e_{2}^{\mathrm{MA}} \geq e_{2}^{\mathrm{MT}}$.

One interesting finding from Corollary 5.3 is that given the conflicting nature of two performance measures, when designing commission rates for the ISP, the brand owner will raise $\beta_{1}$ and lower $\beta_{2}$ to induce higher $e_{1}$ compared to the multi-agent case. However, $e_{1}^{\mathrm{MT}}$ is less than $e_{1}^{\mathrm{MA}}$, so the ISP still refrains from exerting more sales effort. Now we find that both effort levels are higher under the multi-agent case, and since effort levels derived in the decentralized models will always be less than the first-best solutions for the centralized case $e_{i}^{C}(i=1,2)$ regardless of the outsourcing model due to the agents' risk aversion, the difference in profit under the two cases can be well explained because higher effort levels result in more profit earned by the firm. A natural question that arises is why the firm cannot motivate the agent to exert as much effort under the multi-task case as under the multi-agent case. We believe that due to its inflexibility in setting commission rates, the firm will incur more costs in the trade-off of inducing higher effort levels and protecting agents from risk taking by paying a premium. In fact, suppose that we can adjust the commission rates under multi-task 
case in such a way that the corresponding effort levels are the same as those under multi-agent case; then, the brand owner will need to pay a higher risk premium, as shown in the corollary below.

Corollary 5.4. If the firm offers $\beta_{i}=\tilde{\beta}_{i}^{\mathrm{MT}}$ to the ISP under the multi-task case such that $e_{i}=e_{i}^{\mathrm{MA}}$ will be chosen, then $\operatorname{RP}\left(\widetilde{\beta}_{1}^{\mathrm{MT}}, \widetilde{\beta}_{2}^{\mathrm{MT}}\right)>\operatorname{RP}\left(\beta_{1}^{\mathrm{MA}}, \beta_{2}^{\mathrm{MA}}\right)$, where $\operatorname{RP}\left(\beta_{1}, \beta_{2}\right)=\frac{1}{2} \rho\left(\beta_{1}^{2} \sigma^{2}+\beta_{2}^{2} \sigma_{m}^{2}\right)$ is the risk premium as a function of $\left(\beta_{1}, \beta_{2}\right)$.

This result implies that considering the higher potential risk premium to be paid, the brand owner will adjust commission rates, and so effort levels will decrease. This finding again provides sound evidence that the firm suffers from inflexibility in setting contract parameters under the multi-task case and thus earns less profit.

We have shown how the gap in effort levels between two outsourcing models results in a profit difference, given that all problem parameters are fixed. Next we will investigate this relationship from a dynamic perspective. Specifically, we compare the changing rate in CSI measure accuracy of the brand owner's profits under different scenarios, i.e., $\frac{\partial \pi^{i}}{\partial n}, i \in\{\mathrm{MT}, \mathrm{MA}\}$, as shown by the proposition below.

Proposition 5.5. The brand owner benefits more from increasing CSI measure accuracy under the multi-agent case than the multi-task case, i.e., $-\frac{\partial \pi^{\mathrm{MA}}}{\partial n}>-\frac{\partial \pi^{\mathrm{MT}}}{\partial n}$. However, the benefit vanishes as the CSI measure error becomes extremely large.

Now we see that the CSI measure accuracy critically affects the profit earned under different outsourcing models and compensation plans. When it is too difficult to measure CS precisely, the profit difference under the two scenarios is indeed not noticeable. But increasing measure accuracy is always more effective in generating extra profit under the multi-agent case. So when the firm can gradually increase the measure accuracy, this advantage will accumulate and enlarge the profit gap, and thus, the dominant relationship between the two scenarios is well explained. In fact, we can further show that the changing rates in $n$ for $e_{i}^{\mathrm{MT}}$ and $e_{i}^{\mathrm{MA}}, i=1,2$ follow a similar path, as shown by the proposition below.

Proposition 5.6. When CSI can be measure more accurately, the corresponding changes in both effort levels are more significant under the multi-agent case than the multi-task case, i.e., $-\frac{\partial e_{1}^{\mathrm{MA}}}{\partial n}>-\frac{\partial e_{1}^{\mathrm{MT}}}{\partial n}, \lim _{n \rightarrow \infty} e_{1}^{\mathrm{MA}}=$ $\lim _{n \rightarrow \infty} e_{1}^{\mathrm{MT}}=\frac{1-\theta}{k^{2} \rho \sigma^{2}+k} . \quad-\frac{\partial e_{2}^{\mathrm{MA}}}{\partial n}>-\frac{\partial e_{2}^{\mathrm{MT}}}{\partial n}, \lim _{n \rightarrow \infty} e_{2}^{\mathrm{MA}}=\lim _{n \rightarrow \infty} e_{2}^{\mathrm{MT}}=0$.

The proposition states that the difference in effort levels under the two scenarios will also vanish when $n$ is very high, while the gaps between them enlarge as the CSI measure becomes more accurate. Thus, the results in Proposition 5.5 can be directly derived with the changing pattern of effort levels with CSI measure accuracy.

We have found the profit difference for different outsourcing models and explored its underlying reasons from several perspectives. Next, we will numerically show the relative magnitude of this difference under certain parameter settings. Denote $\delta \tilde{\pi}=\pi^{\mathrm{MA}}-\pi^{\mathrm{MT}}, \Delta \tilde{\pi}=\delta \tilde{\pi} / \pi^{\mathrm{MT}}$, and Figure 3 shows how $\Delta \tilde{\pi}$ varies with certain model parameters. We observe that the relative differences are decreasing in $n$ and unimodal in $\theta$ and $m$, and the profit difference can be very significant, surpassing $100 \%$ for some specific parameter settings. Note that the business outsourcing model is usually chosen before compensation plans are determined and are treated as an exogenous system factor in our paper, so our main conclusion from our analytical and numerical analysis is that if CS is the major performance measure of the agent's quality-control effort level, then the firm operating under the multi-agent scenario will receive a greater benefit than it will under the multi-task scenario, and this profit difference can be more significant when CS is measured more accurately. Of course, if we suppose that outsourcing model choice can be the firm's endogenous decision, then in our setting, contracting with separate service providers is preferable to contracting with a single agent.

\section{Conclusion AND FUture Research}

In this paper, we consider the incentive design problem for a brand owner that delegates its online marketing and operational business lines to service providers. Two representative scenarios that are summarized from 

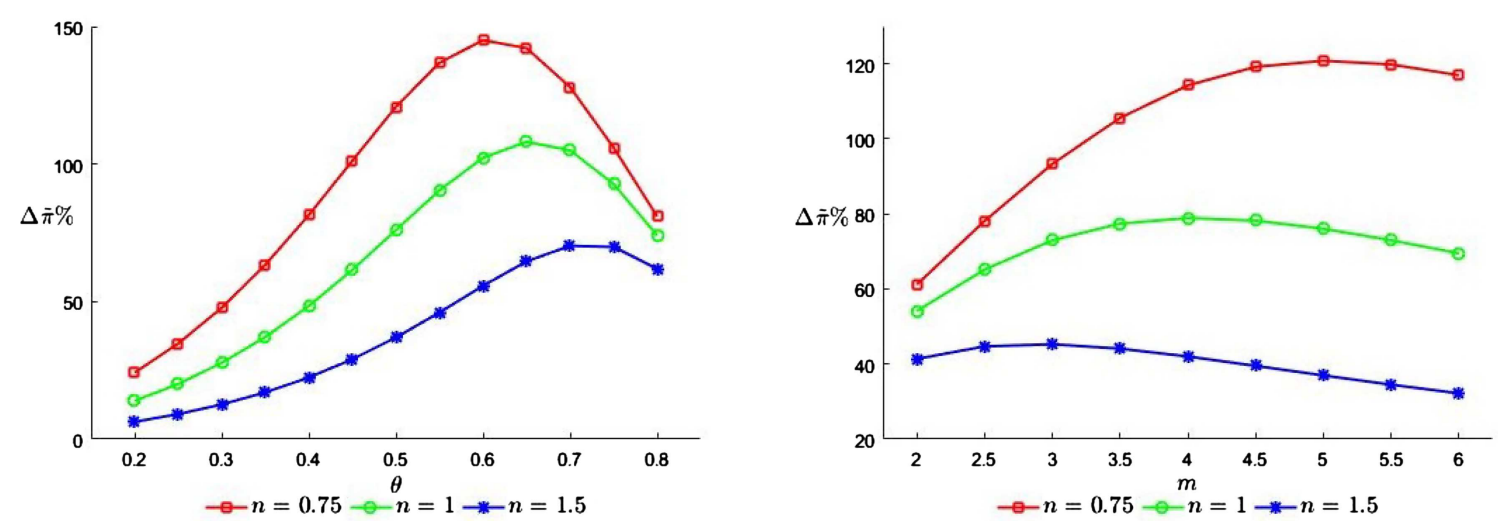

FiguRE 3. Relative profit difference varying with $\theta$ (left) and $m$ (right), with $n=0.75,1,1.5$ and base parametric values $\theta=0.5, m=5, k=2, \rho=2, \sigma=3$.

some widely observed business models in the e-business practice, i.e., the multi-task and multi-agent cases, are studied. By innovatively employing the disconfirmation model of CS, we connect CS with sales and qualitycontrol efforts, and formulate the CS-based contract design problems following the principal-agent framework.

We derive the optimal contract parameters, the agent(s)' effort levels and the brand owner's profits in closed form under both scenarios. Through extensive sensitivity analyses, we find that increasing CSI measure accuracy is beneficial for the brand owner, while the magnitude of benefit may depend on certain outsourcing models and problem parameters. Specifically, the benefit is commonly more substantial when the marginal cost of qualitycontrol effort is relatively smaller than the marginal cost of sales effort, while the influence of the disconfirmation factor on the benefit is subtler and varies in different outsourcing models. We conduct an in-depth investigation on the profit difference between scenarios and its underlying driving force. An interesting finding is that the brand owner always earns more profit under the multi-agent case than under the multi-task case. The profit gap becomes more significant when the CSI is measured with more accuracy and can even be expanded when the marketing service provider is compensated on both sales and CSI under the multi-agent case. This observation is attributed mainly to the fact that, the firm in the multi-task case faces more inflexibility in setting commission rates to reach a balance between inducing a high effort level and providing enough risk premium to the single agent. While under the multi-agent case, the firm enjoys more degrees of freedom to manipulate the agents' behavior. From a practical perspective, our work also suggests several insightful points. First, when it is cost effective, firms should take all means to increase the CSI measure accuracy and pay attention to other system factors that can considerably influence the associated benefit. Second, when it is possible for the firm to choose outsourcing models, then under our setting, it is preferable to delegate business lines to separate service providers than to contract with a single one.

Our paper is among the first to integrate the disconfirmation model of CS and CS-based incentive design problems. Due to the importance of CS in marketing theory and practice, we believe that research opportunities abound. Our paper is featured with some stylized assumptions and settings, which on one hand facilitate clear and insightful results, whereas on the other hand make the conclusions restricted by model conditions, and leaves spaces for several avenues for future research. First, some assumptions in our model, such as that the cost-related parameters $\left(k_{1}\right.$ and $\left.k_{2}\right)$ are the same in both multi-task and multi-agent scenarios, that both the MSP and OSP have the same risk attitude in the multi-agent case, and that the functions composing customer satisfaction $\left(f_{1}\right.$ and $\left.f_{2}\right)$ are both linear, may be somewhat strict. We want to consider the relaxation of them and check whether our analytical results may be preserved or changed under more generalized settings. $t$ will also be interesting to incorporate other issues discussed in principal-agent models, such as double moral hazard, other contract forms (e.g., quota-bonus contracts) and information asymmetry, into our model. In Appendix B, 
we present a preliminary exploration on our incentive design problems when some of our key assumptions are generalized, and identify the resemblances and differences of the results from those derived in our paper. Second, we assume that demand is affected mainly by sales and quality-control efforts, so a possible extension is to include other factors such as product price into the demand model and to consider incentive design problems in this setting. Finally, our model captures the inter-temporal effect of CS in a two-period model. But with all decisions made simultaneously, it still falls in a static framework. Thus it will be worthwhile to consider a generalized multi-period model where the decisions of of the brand owner and agents interact with each other dynamically. The corresponding analysis, however, can be more challenging and new methodologies like dynamic programming may be applied to discover more insightful analytical results in that case.

\section{Appendix A. Proofs}

Proof of Lemma 2.1. It is straightforward to show

$$
\bar{m}-\underline{m}=\frac{(\theta+1)^{2}\left(\theta+n^{2}\left(k \rho \sigma^{2}+1\right)\right)}{(1-\theta) \theta k \rho \sigma^{2}\left(\theta^{2}+n^{2}\right)}>0 .
$$

From

$$
\frac{\partial \bar{m}}{\partial \theta}=\frac{(\theta+1)(3 \theta-1)\left(k \rho \sigma^{2}+1\right)}{(\theta-1)^{2} \theta^{2} k \rho \sigma^{2}},
$$

we can see $\bar{m}$ crosses 0 upwards only once and at $\theta=1 / 3$, where its minimum is reached, the corresponding functional value can be derived. For $\underline{m}$, we have

$$
\frac{\partial \underline{m}}{\partial \theta}=\frac{(\theta+1)\left(k \rho \sigma^{2}\left(\theta^{2}(3 \theta-1)+(\theta(3-2 \theta)+1) n^{2}\right)+2(\theta-1)^{2}\left(\theta-n^{2}\right)\right)}{(\theta-1)^{2} k \rho \sigma^{2}\left(\theta^{2}+n^{2}\right)^{2}},
$$

and we focus only on part of the numerator $k \rho \sigma^{2}\left(\theta^{2}(3 \theta-1)+(\theta(3-2 \theta)+1) n^{2}\right)+2(\theta-1)^{2}\left(\theta-n^{2}\right)$, which is cubic in $\theta$, and we denote it as $f(\theta)$ (here, we define the domain of $f(\theta)$ as the whole real line, not limited to $(0,1))$. First, note that its third-order derivative in $\theta$ is $18 k \rho \sigma^{2}+12>0$, so $f(\theta)$ will first be concave then convex and will either be increasing in $\theta$ (which corresponds to the case $f^{\prime}(\theta) \geq 0$ ) or first increasing then decreasing and then increasing again (which corresponds to the case $f^{\prime}(\theta)$ crosses 0 twice). Notice that $\underline{m}(\theta)=-\frac{1}{k n^{2} \rho \sigma^{2}}<0$ when $\theta=0$, so it suffices to show that $f(\theta)$ is positive or crosses 0 upwards only once, since the former case indicates that $\underline{m}(\theta)$ is increasing in $\theta$, while the latter case implies that $\underline{m}^{+}(\theta)$ is increasing. Both cases will make the monotonicity of $\underline{m}^{+}(\theta)$ in $\theta$ hold.

Now, we have $f(1)=2 k\left(n^{2}+1\right) \rho \sigma^{2}>0, f(0)=n^{2}\left(k \rho \sigma^{2}-2\right), f^{\prime}(0)=n^{2}\left(3 k \rho \sigma^{2}+4\right)+2>0, f^{\prime}(1)=$ $-k\left(n^{2}-7\right) \rho \sigma^{2}, f^{\prime \prime}(0)=-2\left(2 n^{2}+1\right) \rho \sigma^{2}-4\left(n^{2}+2\right)<0, f^{\prime \prime}(1)=-4\left(n^{2}-4\right) \rho \sigma^{2}-4 n^{2}+4$. Since $f^{\prime \prime}(1)$ is decreasing in $n^{2}$ and when $n^{2} \geq \frac{4 k \rho \sigma^{2}+1}{k \rho \sigma^{2}+1}, f^{\prime \prime}(1) \leq 0$, we can infer from the properties of $f(\theta)$ (first concave then convex) that when $n^{2} \geq \frac{4 k \rho \sigma^{2}+1}{k \rho \sigma^{2}+1}, f(\theta)$ will always be concave and will be either positive (when $k \rho \sigma^{2} \geq 2$ ) or cross 0 upwards only once (when $k \rho \sigma^{2}<2$ ). These two cases imply the sufficient condition we derive above.

Since $\frac{4 k \rho \sigma^{2}+1}{k \rho \sigma^{2}+1}>1>1 / 2$, now consider the case when $n^{2} \in\left[1 / 2, \frac{4 k \rho \sigma^{2}+1}{k \rho \sigma^{2}+1}\right]$. First, consider the subcase when $f(\theta)$ is increasing, i.e., when the discriminant of $f^{\prime}(\theta)$ in $\theta$ is negative (this is sufficient since $f^{\prime \prime \prime}(\theta)>0$ ). Notice that the discriminant is

$$
4 k^{2}\left(4 n^{4}-23 n^{2}+1\right) \rho^{2} \sigma^{4}+8 k\left(4 n^{4}-17 n^{2}-5\right) \rho \sigma^{2}+16,\left(n^{2}-1\right)^{2}
$$

a quadratic and convex function in $n^{2}$. As a function of $n^{2}$, this discriminant can be shown to be convex in $n^{2}$ and is equal to $-\frac{36 k \rho \sigma^{2}\left(k \rho \sigma^{2}+2\right)\left(3 k \rho \sigma^{2}+2\right)}{k \rho \sigma^{2}+1}<0$ when $n^{2}=\frac{4 k \rho \sigma^{2}+1}{k \rho \sigma^{2}+1}$ and equal to $4-2 k \rho \sigma^{2}\left(19 k \rho \sigma^{2}+50\right)$ when $n^{2}=1 / 2$. It is easy to show that $4-2 x(19 x+50)$ is decreasing in positive $x$, so now, when $k \rho \sigma^{2} \geq \frac{1}{19}(\sqrt{663}-25)$, 
i.e., $4-2 k \rho \sigma^{2}\left(19 k \rho \sigma^{2}+50\right) \leq 0$, then the discriminant above is always negative, and thus, $f(\theta)$ is increasing. Therefore, either the single-crossing property will be satisfied (when $k \rho \sigma^{2} \in\left[\frac{1}{19}(\sqrt{663}-25)\right.$, 2$]$ ), or if $f(\theta) \geq 0$ (when $k \rho \sigma^{2} \geq 2$ ), then the sufficient condition is also satisfied.

We still have to consider the case when $n^{2} \in\left[1 / 2, \frac{4 k \rho \sigma^{2}+1}{k \rho \sigma^{2}+1}\right]$ and $k \rho \sigma^{2}<\frac{1}{19}(\sqrt{663}-25)$ (notice $f(0)<0$ in this case), where the determinant of $f^{\prime}(\theta)$ in $\theta$ is positive and so $f(\theta)$ will be concave first and then convex. From $f^{\prime}(0)>0, f^{\prime}(1)>0$, we now conclude that $f(\theta)$ is first increasing and then decreasing and then increasing again. Since $\frac{4 x+1}{x+1}$ is increasing in $x$, the possible maximum of $n^{2}$ will be $\frac{1}{11}(38-\sqrt{663}) \approx 1.11$. Note that if we can show that the single local minimum of $f(\theta)$ is positive, then the function must satisfy the single-crossing property. A sufficient condition for this to hold is that $f(\theta)>0 \forall \theta \in[\tilde{\theta}, 1]$, where $\tilde{\theta}=\frac{1}{9}\left(\frac{2\left(n^{2}+5\right)}{3 k \rho \sigma^{2}+2}+2 n^{2}+1\right)$ is the point where $f(\theta)$ turns from concave to convex and can be derived by solving $f^{\prime \prime}(\theta)=0$, since at the local minimum, $f(\theta)$ must be convex. Now, notice that $\tilde{\theta}$ is increasing in $n^{2}$ and decreasing in $k \rho \sigma^{2}$, so its minimum $\frac{1}{66}(\sqrt{663}+27) \approx 0.8$ can be reached when $n^{2}=1 / 2, k \rho \sigma^{2}=\frac{1}{19}(\sqrt{663}-25)$.

Now, consider the value of

$$
f(\theta)=k \rho \sigma^{2}\left(\theta^{2}(3 \theta-1)+(\theta(3-2 \theta)+1) n^{2}\right)+2(\theta-1)^{2}\left(\theta-n^{2}\right)
$$

when $\theta \in[\tilde{\theta}, 1]$. It is easy to find that one factor of the first term, $\theta^{2}(3 \theta-1)+(\theta(3-2 \theta)+1) n^{2}$, is positive when $\theta \in\left[\frac{1}{66}(\sqrt{663}+27), 1\right], n^{2} \in\left[1 / 2, \frac{1}{11}(38-\sqrt{663})\right]$, given the positivity of $3 \theta-1$ and $3-2 \theta$. Consider the derivative in $\theta$ of this factor, $\theta(9 \theta-2)+(3-4 \theta) n^{2}$, where the first term is positive and the second is negative. The minimum of $\theta(9 \theta-2)$ approximates 4.15 , while the minimum of $(3-4 \theta) n^{2}$ approximates -1.11 , so the derivative will always be positive. Therefore, this factor is both positive and increasing in $\theta$. Now, when the term $2(\theta-1)^{2}\left(\theta-n^{2}\right) \geq 0$, then $f(\theta) \geq 0$ is shown; otherwise, we can show it is increasing in $\theta$ when $2(\theta-1)^{2}\left(\theta-n^{2}\right)<0$, thus $f(\theta)$ is also increasing because when $2(\theta-1)^{2}\left(\theta-n^{2}\right)<0$, i.e., $n^{2}>\theta$, its derivative in $\theta$ is $(\theta-1)\left(3 \theta-2 n^{2}-1\right)>(\theta-1)(\theta-1)>0$. So for the latter case, it suffices to show $f(\tilde{\theta}) \geq 0$.

Now, let $K=k \rho \sigma^{2}$, and we have

$$
\begin{aligned}
f(\tilde{\theta})= & \frac{-2 K^{3}+30 K^{2}-16(K+1)^{3} n^{6}+6(K+1)(K(23 K+34)+8) n^{4}}{27(3 K+2)^{2}} \\
& +\frac{12(K(K(26 K+27)+6)-4) n^{2}+156 K+16}{27(3 K+2)^{2}} .
\end{aligned}
$$

We see it is equivalent to show that the denominator is positive, while the derivative of the numerator in $K$ equals

$$
6\left(-K^{2}-8(K+1)^{2} n^{6}+3(K(23 K+38)+14) n^{4}+12(K(13 K+9)+1) n^{2}+10 K+26\right) .
$$

Note that the term $-K^{2}-8(K+1)^{2} n^{6}$ is decreasing in $n$ and $K$ and reaches its minimum $-\frac{8(4 \sqrt{663}-81)^{3}}{6859}-$ $\frac{1}{361}(\sqrt{663}-25)^{2} \approx-12.4$ when $n$ and $K$ are as large as possible; so we have $-K^{2}-8(K+1)^{2} n^{6}<26$ and the derivative above is positive. Thus, the numerator is increasing in $K$ with its minimum $-16\left(n^{2}-1\right)^{3}$ at $K=0$. Note that when $K=0, n^{2} \in[1 / 2,1]$, the minimum is positive, so the numerator and $f(\tilde{\theta})$ must be positive.

Summarizing all cases above, we can conclude that $f(\theta)$ will either be positive or have the single-crossing property, which ensures that $\max \{\underline{m}, 0\}$ is increasing in $\theta$. Now, by incorporating the quasi-convexity of $\bar{m}$, we can derive that for any $m \leq \frac{8\left(k \rho \sigma^{2}+1\right)}{\rho \sigma^{2}}, m \leq \bar{m}$ always holds, while $m \geq \underline{m}$ when $\theta \leq \underline{m}^{-1}(m)$. Let $\bar{\theta}(m)=\underline{m}^{-1}(m)$; then, we can prove that $m$ is feasible when $\theta \in[0, \bar{\theta}(m)]$. 
Proof of Theorem 3.1. By solving constraint (2), we have $e_{1}^{*}=\frac{\beta_{1}-\beta_{2} \theta}{k_{1}}, e_{2}^{*}=\frac{\beta_{2}(\theta+1)}{k_{2}}$. Substituting this and the binding constraint (3) to $\pi$, we present the profit function as a function of $\beta_{i}(i=1,2)$

$$
\begin{aligned}
\pi\left(\beta_{1}, \beta_{2}\right)= & \frac{-\beta_{1} m\left(\beta_{1}+2 \theta+\beta_{1} k \rho \sigma^{2}-2\right)-\beta_{2}^{2}\left(k m n^{2} \rho \sigma^{2}+\theta(\theta+\theta m+2)+1\right)}{2 k m} \\
& +\frac{2 \beta_{2}\left(\beta_{1} \theta m+\theta(\theta+(\theta-1) m+2)+1\right)}{2 k m} .
\end{aligned}
$$

The Hessian matrix is

$$
H=\left[\begin{array}{cc}
-\rho \sigma^{2}-\frac{1}{k} & \frac{\theta}{k} \\
\frac{\theta}{k} & -\frac{k m n^{2} \rho \sigma^{2}+\theta(\theta+\theta m+2)+1}{k m}
\end{array}\right],
$$

and can be shown negative definite so $\pi\left(\beta_{1}, \beta_{2}\right)$ is concave. The optimal solutions are easily derived by solving the first-order conditions (FOC) and variable substitution.

Proof of Proposition 3.2. First, we have

$$
\begin{aligned}
\frac{\partial \beta_{1}^{\mathrm{MT}}}{\partial m} & =-\frac{\theta(\theta+1)^{2} k \rho \sigma^{2}\left(\theta+n^{2}\left(k \rho \sigma^{2}+1\right)\right)}{\left((\theta+1)^{2}+k^{2} m n^{2} \rho^{2} \sigma^{4}+k \rho \sigma^{2}\left((\theta+1)^{2}+m\left(\theta^{2}+n^{2}\right)\right)\right)^{2}}<0, \\
\frac{\partial \beta_{1}^{\mathrm{MT}}}{\partial n} & =\frac{2 \theta k m n \rho \sigma^{2}\left(-(\theta+1)^{2}-k \rho \sigma^{2}(\theta(\theta+(\theta-1) m+2)+1)\right)}{\left((\theta+1)^{2}+k^{2} m n^{2} \rho^{2} \sigma^{4}+k \rho \sigma^{2}\left((\theta+1)^{2}+m\left(\theta^{2}+n^{2}\right)\right)\right)^{2}}, \\
& =-\frac{2 \theta k m n \rho \sigma^{2} \beta_{2}^{\mathrm{MT}}}{(\theta+1)^{2}+k^{2} m n^{2} \rho^{2} \sigma^{4}+k \rho \sigma^{2}\left((\theta+1)^{2}+m\left(\theta^{2}+n^{2}\right)\right)}<0 \\
\frac{\partial \beta_{1}^{\mathrm{MT}}}{\partial \theta} & =\frac{k m \rho \sigma^{2}\left(-2 \theta(\theta+1)-k m n^{4} \rho \sigma^{2}\left(k \rho \sigma^{2}+1\right)+n^{2}\left(\theta^{2}+k \rho \sigma^{2}\left(\theta^{2}+(\theta-2) \theta m-1\right)-1\right)\right)}{\left((\theta+1)^{2}+k^{2} m n^{2} \rho^{2} \sigma^{4}+k \rho \sigma^{2}\left((\theta+1)^{2}+m\left(\theta^{2}+n^{2}\right)\right)\right)^{2}}<0,
\end{aligned}
$$

where the last inequality holds since $\theta^{2}+k \rho \sigma^{2}\left(\theta^{2}+(\theta-2) \theta m-1\right)-1<0$ due to $\theta<1$. We also have

$$
\begin{aligned}
& \frac{\partial \beta_{1}^{\mathrm{MT}}}{\partial k}=\frac{\rho \sigma^{2}\left((\theta-1) k^{2} m^{2} n^{4} \rho^{2} \sigma^{4}-2(\theta+1)^{2} k m n^{2} \rho \sigma^{2}-(\theta+1)^{2}\left(\theta\left(\theta+m\left(\theta+n^{2}\right)+2\right)+1\right)\right)}{\left((\theta+1)^{2}+k^{2} m n^{2} \rho^{2} \sigma^{4}+k \rho \sigma^{2}\left((\theta+1)^{2}+m\left(\theta^{2}+n^{2}\right)\right)\right)^{2}}<0, \\
& \frac{\partial \beta_{1}^{\mathrm{MT}}}{\partial \rho}=\frac{k}{\rho} \frac{\partial \beta_{1}^{\mathrm{MT}}}{\partial k}<0, \quad \frac{\partial \beta_{1}^{\mathrm{MT}}}{\partial \sigma}=\frac{2 k}{\sigma} \frac{\partial \beta_{1}^{\mathrm{MT}}}{\partial \rho}<0 .
\end{aligned}
$$

For $\beta_{2}^{\mathrm{MT}}$, we have

$$
\begin{aligned}
\frac{\partial \beta_{2}^{\mathrm{MT}}}{\partial m} & =-\frac{(\theta+1)^{2} k \rho \sigma^{2}\left(k \rho \sigma^{2}+1\right)\left(\theta+n^{2}\left(k \rho \sigma^{2}+1\right)\right)}{\left((\theta+1)^{2}+k^{2} m n^{2} \rho^{2} \sigma^{4}+k \rho \sigma^{2}\left((\theta+1)^{2}+m\left(\theta^{2}+n^{2}\right)\right)\right)^{2}}<0, \\
\frac{\partial \beta_{2}^{\mathrm{MT}}}{\partial n} & =\frac{k \rho \sigma^{2}+1}{\theta} \frac{\partial \beta_{1}^{\mathrm{MT}}}{\partial n}<0, \\
\frac{\partial \beta_{2}^{\mathrm{MT}}}{\partial k} & =\frac{m \rho \sigma^{2}\left(-k^{2} n^{2} \rho^{2} \sigma^{4}(\theta(\theta+(\theta-1) m+2)+1)-2(\theta+1)^{2} k n^{2} \rho \sigma^{2}-(\theta+1)^{2}\left(\theta+n^{2}\right)\right)}{\left((\theta+1)^{2}+k^{2} m n^{2} \rho^{2} \sigma^{4}+k \rho \sigma^{2}\left((\theta+1)^{2}+m\left(\theta^{2}+n^{2}\right)\right)\right)^{2}} .
\end{aligned}
$$

Note that $\frac{\partial \beta_{2}^{\mathrm{MT}}}{\partial m}=0$ when $m=\bar{m}$, so with its monotonicity in $m$ and our assumption on $m, \beta_{2}^{\mathrm{MT}}>0$ holds. For $\frac{\partial \beta_{2}^{\mathrm{MT}}}{\partial k}$, consider part of its numerator

$$
-k^{2} n^{2} \rho^{2} \sigma^{4}(\theta(\theta+(\theta-1) m+2)+1)-2(\theta+1)^{2} k n^{2} \rho \sigma^{2}-(\theta+1)^{2}\left(\theta+n^{2}\right),
$$


whose derivative in $m$ equals $(1-\theta) \theta k^{2} n^{2} \rho^{2} \sigma^{4}>0$, so this part is increasing in $m$. We further find that when $m=\bar{m}$, this part equals $-(\theta+1)^{2}\left(\theta+n^{2}\left(k \rho \sigma^{2}+1\right)\right)<0$, so in the feasible region of $m$, the part above will always be negative; thus, $\frac{\partial \beta_{2}^{\mathrm{MT}}}{\partial k}<0$. We can also derive $\frac{\partial \beta_{2}^{\mathrm{MT}}}{\partial \rho}=\frac{k}{\rho} \frac{\partial \beta_{2}^{\mathrm{MT}}}{\partial k}<0, \frac{\partial \beta_{2}^{\mathrm{MT}}}{\partial \sigma}=\frac{2 k}{\sigma} \frac{\partial \beta_{2}^{\mathrm{MT}}}{\partial k}<0$.

For

$$
\frac{\partial \beta_{2}^{\mathrm{MT}}}{\partial \theta}=\frac{k m \rho \sigma^{2}\left(\theta^{2}+k \rho \sigma^{2}\left(\theta^{2}(m+1)-1\right)+n^{2}\left(k \rho \sigma^{2}+1\right)\left(2(\theta+1)+k \rho \sigma^{2}(2 \theta(m+1)-m+2)\right)-1\right)}{\left((\theta+1)^{2}+k^{2} m n^{2} \rho^{2} \sigma^{4}+k \rho \sigma^{2}\left((\theta+1)^{2}+m\left(\theta^{2}+n^{2}\right)\right)\right)^{2}},
$$

consider part of its numerator

$$
\theta^{2}+k \rho \sigma^{2}\left(\theta^{2}(m+1)-1\right)+n^{2}\left(k \rho \sigma^{2}+1\right)\left(2(\theta+1)+k \rho \sigma^{2}(2 \theta(m+1)-m+2)\right)-1
$$

whose derivative in $m$ is $k \rho \sigma^{2}\left(\theta^{2}+(2 \theta-1) n^{2}\left(k \rho \sigma^{2}+1\right)\right)$. So when $\theta \geq 1 / 2$, this derivative is positive and the numerator will be increasing in $m$. Now, at $m=0$, the value of the numerator is $(\theta+1)$ $\left(k \rho \sigma^{2}+1\right)\left(\theta+2 n^{2}\left(k \rho \sigma^{2}+1\right)-1\right)>0$ due to $n^{2} \geq 1 / 2$, so the numerator and, thus, $\frac{\partial \beta_{2}^{\mathrm{MT}}}{\partial \theta}$ will be positive.

We can also consider the derivative in $\theta$ of the numerator, $2\left(k(m+1) \rho \sigma^{2}+1\right)\left(\theta+n^{2}\left(k \rho \sigma^{2}+1\right)\right)>0$, which implies that $\frac{\partial \beta_{2}^{\mathrm{MT}}}{\partial \theta}$ will either be positive or negative or cross 0 upwards only once. Therefore, $\beta_{2}^{\mathrm{MT}}$ is quasi-convex in $\theta$. Now, when $\theta=0$, the value of the numerator is $n^{2}\left(k \rho \sigma^{2}+1\right)\left(2-k(m-2) \rho \sigma^{2}\right)-k \rho \sigma^{2}-1$, which is decreasing in $m$. When $m<\frac{2 n^{2}-1}{k n^{2} \rho \sigma^{2}}+2$, this value is positive, which implies the positivity of the numerator and $\frac{\partial \beta_{2}^{\mathrm{MT}}}{\partial \theta}$.

From the expression of $\beta_{1}^{\mathrm{MT}}$, we can see its positivity with ease, and $e_{2}^{\mathrm{MT}}>0$ is straightforward since $e_{2}^{*}=\frac{\beta_{2}^{\mathrm{MT}}(\theta+1)}{k_{2}}$, while we have shown $\beta_{2}^{\mathrm{MT}}>0$. To show $e_{1}^{\mathrm{MT}} \geq 0$, note

$$
\frac{\partial e_{1}^{\mathrm{MT}}}{\partial m}=\frac{\theta(\theta+1)^{2} k \rho^{2} \sigma^{4}\left(\theta+n^{2}\left(k \rho \sigma^{2}+1\right)\right)}{\left((\theta+1)^{2}+k^{2} m n^{2} \rho^{2} \sigma^{4}+k \rho \sigma^{2}\left((\theta+1)^{2}+m\left(\theta^{2}+n^{2}\right)\right)\right)^{2}}>0,
$$

and $e_{1}^{\mathrm{MT}}=0$ when $m=\underline{m}$. Since we have assumed $m>\underline{m}^{+}, e_{1}^{\mathrm{MT}} \geq 0$ holds. The relationship between $e_{i}^{\mathrm{MT}}$ and $e_{i}^{C}, i=1,2$ can be easily derived, as shown below:

$$
\begin{aligned}
& e_{1}^{C}-e_{1}^{\mathrm{MT}}=\frac{\rho \sigma^{2}\left((\theta+1)^{2}+(1-\theta) k m n^{2} \rho \sigma^{2}\right)}{(\theta+1)^{2}+k^{2} m n^{2} \rho^{2} \sigma^{4}+k \rho \sigma^{2}\left((\theta+1)^{2}+m\left(\theta^{2}+n^{2}\right)\right)}>0, \\
& e_{2}^{C}-e_{2}^{\mathrm{MT}}=\frac{(\theta+1) \rho \sigma^{2}\left(\theta+n^{2}\left(k \rho \sigma^{2}+1\right)\right)}{(\theta+1)^{2}+k^{2} m n^{2} \rho^{2} \sigma^{4}+k \rho \sigma^{2}\left((\theta+1)^{2}+m\left(\theta^{2}+n^{2}\right)\right)}>0 .
\end{aligned}
$$

For the brand owner's optimal profit $\pi^{\mathrm{MT}}$, we have

$$
\begin{aligned}
\frac{\partial \pi^{\mathrm{MT}}}{\partial n} & =-\frac{n \rho\left((\theta+1)^{2} \sigma+k \rho \sigma^{3}(\theta(\theta+(\theta-1) m+2)+1)\right)^{2}}{\left((\theta+1)^{2}+k^{2} m n^{2} \rho^{2} \sigma^{4}+k \rho \sigma^{2}\left((\theta+1)^{2}+m\left(\theta^{2}+n^{2}\right)\right)\right)^{2}}<0, \\
\frac{\partial \pi^{\mathrm{MT}}}{\partial m} & =-\frac{(\theta+1)^{2}\left((\theta+1)^{2}+2 k^{2} m n^{2} \rho^{2} \sigma^{4}+k \rho \sigma^{2}\left((\theta+1)^{2}+m\left(\theta^{2}+\theta+2 n^{2}\right)\right)\right)}{2 k m^{2}\left((\theta+1)^{2}+k^{2} m n^{2} \rho^{2} \sigma^{4}+k \rho \sigma^{2}\left((\theta+1)^{2}+m\left(\theta^{2}+n^{2}\right)\right)\right)} \beta_{2}^{\mathrm{MT}}<0,
\end{aligned}
$$

so part (iii) is proven. It can also be shown that

$$
-\frac{\partial^{2} \pi^{\mathrm{MT}}}{\partial n \partial m}=\frac{2(\theta+1)^{2} k n \rho^{2} \sigma^{4}\left(k \rho \sigma^{2}+1\right)\left(\theta+n^{2}\left(k \rho \sigma^{2}+1\right)\right)}{\left((\theta+1)^{2}+k^{2} m n^{2} \rho^{2} \sigma^{4}+k \rho \sigma^{2}\left((\theta+1)^{2}+m\left(\theta^{2}+n^{2}\right)\right)\right)^{2}} \beta_{2}^{\mathrm{MT}}<0 .
$$

Now, since $-\frac{\partial \pi^{\mathrm{MT}}}{\partial n}$ is the marginal profit gain from increasing CS measure accuracy, $-\frac{\partial^{2} \pi^{\mathrm{MT}}}{\partial n \partial m}$ can be viewed as the influence of increasing marginal cost of quality-control effort on this profit gain, so $-\frac{\partial^{2} \pi^{\mathrm{MT}}}{\partial n \partial m}<0$ implies that 
increasing CSI measure accuracy is more effective when the marginal cost of quality-control effort is relatively smaller. We also have

$$
-\frac{\partial^{2} \pi^{\mathrm{MT}}}{\partial n \partial \theta}=2 n \rho \sigma^{2} \beta_{2}^{\mathrm{MT}} \frac{\partial \beta_{2}^{\mathrm{MT}}}{\partial \theta}
$$

which implies that $-\frac{\partial^{2} \pi^{\mathrm{MT}}}{\partial n \partial \theta}$ has the same sign with $\frac{\partial \beta_{2}^{\mathrm{MT}}}{\partial \theta}$, so when $\theta \geq 1 / 2$, this derivative will be positive.

Proof of Corollary 3.3. When $m=0, \Delta \beta^{\mathrm{MT}}=\frac{1}{k \rho \sigma^{2}+1}-1<0$ and when $m=\bar{m}, \Delta \beta^{\mathrm{MT}}=\frac{1-\theta}{k \rho \sigma^{2}+1}>0$ and we can easily solve $m_{t}=\frac{(\theta+1)^{2}}{(1-\theta)\left(\theta+n^{2}\right)}$ by letting $\Delta \beta^{\mathrm{MT}}=0$. Now, we have

$$
\frac{\partial \Delta \beta^{\mathrm{MT}}}{\partial \theta}=-\frac{(\theta+1)\left(-3 \theta+(\theta-3) n^{2}+1\right)}{(\theta-1)^{2}\left(\theta+n^{2}\right)^{2}} .
$$

For part of the numerator, $-3 \theta+(\theta-3) n^{2}+1$, it is decreasing in $n^{2}$ since $\theta<3$ and when $n^{2}=1 / 2$, its value is $\frac{1}{2}(-5 \theta-1)<0$ so this part is always negative, thus implying the positivity of the derivative. The monotonicity of $m_{t}$ in $n$ is easily seen.

Proof of Theorem 4.1. Following a similar procedure in the proof of Theorem 3.1, present the profit as a function of $\beta_{i}(i=1,2)$ :

$$
\pi\left(\beta_{1}, \beta_{2}\right)=-\frac{\beta_{1}^{2}\left(k m \rho \sigma^{2}+m\right)+\beta_{2}\left(\beta_{2}\left(\theta^{2}+2 \theta+k m n^{2} \rho \sigma^{2}+1\right)-2(\theta+1)^{2}\right)+2 \beta_{1}(\theta-1) m}{2 k m} .
$$

The Hessian matrix

$$
H=\left[\begin{array}{cc}
-\frac{k \rho \sigma^{2}+1}{k} & 0 \\
0 & -n^{2} \rho \sigma-\frac{(\theta+1)^{2}}{k m}
\end{array}\right]
$$

is negative definite so the joint concavity is shown and the optimal solutions are easily derived by solving the FOC and variable substitution.

Proof of Proposition 4.2. Most of the results can be derived straightforwardly by taking the respective derivatives, and the corresponding signs are easy to determine, so we omit them for brevity. For the relationship between $\pi^{\mathrm{MA}}$ and $\theta$, first we have

$$
\begin{aligned}
\frac{\partial \pi^{\mathrm{MA}}}{\partial \theta} & =\frac{1}{2 k}\left(\frac{2(\theta-1)}{k \rho \sigma^{2}+1}+\frac{2(\theta+1)^{5}+4(\theta+1)^{3} k m n^{2} \rho \sigma^{2}}{m\left((\theta+1)^{2}+k m n^{2} \rho \sigma^{2}\right)^{2}}\right), \\
\frac{\partial^{2} \pi^{\mathrm{MA}}}{\partial \theta^{2}} & =\frac{1}{k}\left(\frac{(\theta+1)^{6}+6(\theta+1)^{2} k^{2} m^{2} n^{4} \rho^{2} \sigma^{4}+3(\theta+1)^{4} k m n^{2} \rho \sigma^{2}}{m\left((\theta+1)^{2}+k m n^{2} \rho \sigma^{2}\right)^{3}}+\frac{1}{k \rho \sigma^{2}+1}\right)>0,
\end{aligned}
$$

so the convexity can be proven. Note

$$
\left.\frac{\partial \pi^{\mathrm{MA}}}{\partial \theta}\right|_{\theta=0}=\frac{2 k m n^{2} \rho \sigma^{2}+1}{m k\left(k m n^{2} \rho \sigma^{2}+1\right)^{2}}-\frac{1}{k^{2} \rho \sigma^{2}+k}
$$

is decreasing in $m$ and $n$, which can be shown by taking derivatives. Since when $m \rightarrow 0$, this derivative will go to infinity, while when $m$ and $n$ become infinitely large, this derivative will be negative, we can conclude that when $m$ and $n$ are relatively small, then $\left.\frac{\partial \pi^{\mathrm{MA}}}{\partial \theta}\right|_{\theta=0}$ is positive, and because of convexity, $\frac{\partial \pi^{\mathrm{MA}}}{\partial \theta}$ will always 
be positive since it is increasing in $\theta$, thus implying monotonicity. Otherwise, when $\left.\frac{\partial \pi^{\mathrm{MA}}}{\partial \theta}\right|_{\theta=0}<0$, then the convexity implies that $\pi^{\mathrm{MA}}$ will be decreasing first then increasing in $\theta$ when $\theta \in[0,1]$ since

$$
\left.\frac{\partial \pi^{\mathrm{MA}}}{\partial \theta}\right|_{\theta=1}=\frac{16\left(k m n^{2} \rho \sigma^{2}+2\right)}{k m\left(k m n^{2} \rho \sigma^{2}+4\right)^{2}}>0 .
$$

However, due to the constraint between $m$ and $\theta$ described in model assumption, $\pi^{\mathrm{MA}}$ can either be decreasing or decreasing first then increasing depending on the value of $m$ in $[0, \theta(m)]$.

Part (ii) of the proposition is directly from

$$
-\frac{\partial^{2} \pi^{\mathrm{MA}}}{\partial n \partial m}=-\frac{2(\theta+1)^{4} k n^{3} \rho^{2} \sigma^{4}}{\left((\theta+1)^{2}+k m n^{2} \rho \sigma^{2}\right)^{3}}<0, \quad-\frac{\partial^{2} \pi^{\mathrm{MA}}}{\partial n \partial \theta}=\frac{4(\theta+1)^{3} k m n^{3} \rho^{2} \sigma^{4}}{\left((\theta+1)^{2}+k m n^{2} \rho \sigma^{2}\right)^{3}}>0 .
$$

Proof of Theorem 4.3. Following a similar procedure as that for the proof of Theorem 3.1, present the profit as a function of $\beta_{i}(i=1,2)$ :

$$
\begin{aligned}
\pi\left(\beta_{1}, \gamma, \beta_{2}\right)= & -\frac{-2 \beta_{2}(\theta+1)^{2}+\beta_{1}^{2}\left(k m \rho \sigma^{2}+m\right)+\beta_{2}^{2}\left(\theta^{2}+2 \theta+k m n^{2} \rho \sigma^{2}+1\right)}{2 k m} \\
& -\frac{\gamma_{1} m\left(\gamma_{1}\left(\theta^{2}+k n^{2} \rho \sigma^{2}\right)-2(\theta-1) \theta\right)-2 \beta_{1} m\left(\gamma_{1} \theta-\theta+1\right)}{2 k m} .
\end{aligned}
$$

The Hessian matrix is

$$
H=\left[\begin{array}{ccc}
-\frac{k \rho \sigma^{2}+1}{k} & \frac{\theta}{k} & 0 \\
\frac{\theta}{k} & -\frac{\theta^{2}+k n^{2} \rho \sigma^{2}}{k} & 0 \\
0 & 0 & -\frac{(\theta+1)^{2}}{k m}-n^{2} \rho \sigma^{2}
\end{array}\right],
$$

which is negative definite, so the joint concavity is shown, and the optimal solutions are easily derived by solving the FOC and variable substitution.

Proof of Proposition 4.4. Most of the results can be derived straightforwardly by taking respective derivatives, and the corresponding signs are easy to determine, so we omit them for brevity. For the relationship between $e_{1}^{\mathrm{MAS}}$ and $n$, note

$$
\frac{\partial e_{1}^{\mathrm{MAS}}}{\partial n}=-\frac{k n^{2} \rho \sigma^{2}\left(\theta(3 \theta-2)+n^{2}\right)+\left(\theta^{2}+n^{2}\right)^{2}}{k\left(\theta^{2}+n^{2}\left(k \rho \sigma^{2}+1\right)\right)^{2}} .
$$

Part of the numerator $\theta(3 \theta-2)$ reaches its minimum $-1 / 3$ at $\theta=1 / 3$, while $n^{2}>1 / 2$, so the numerator is positive and the derivative is negative.

For the relationship between $\gamma^{\text {MAS }}$ and $\theta$, note

$$
\frac{\partial \gamma^{\mathrm{MAS}}}{\partial \theta}=\frac{\theta^{2}+(2 \theta-1) n^{2}\left(k \rho \sigma^{2}+1\right)}{\left(\theta^{2}+n^{2}\left(k \rho \sigma^{2}+1\right)\right)^{2}} .
$$

Its numerator is increasing in $\theta$ with a negative value at $\theta=0$ and a positive value at $\theta=1$, so we can conclude that $\gamma^{\mathrm{MAS}}$ is quasi-convex in $\theta$.

For the relationship between $\pi^{\mathrm{MAS}}$ and $\theta$, note

$$
\frac{\partial \pi^{\mathrm{MAS}}}{\partial \theta}=\frac{(\theta+1)^{5}+2(\theta+1)^{3} k m n^{2} \rho \sigma^{2}}{m k\left((\theta+1)^{2}+k m n^{2} \rho \sigma^{2}\right)^{2}}+\frac{(\theta-1)\left(k n^{2} \rho \sigma^{2}\left(\theta(2 \theta-1)+n^{2}\right)+\left(\theta^{2}+n^{2}\right)^{2}\right)}{k\left(\theta^{2}+n^{2}\left(k \rho \sigma^{2}+1\right)\right)^{2}} .
$$


The first term is increasing in $\theta$ since its derivative in $\theta$ is

$$
\frac{(\theta+1)^{6}+6(\theta+1)^{2} k^{2} m^{2} n^{4} \rho^{2} \sigma^{4}+3(\theta+1)^{4} k m n^{2} \rho \sigma^{2}}{k m\left((\theta+1)^{2}+k m n^{2} \rho \sigma^{2}\right)^{3}}>0,
$$

and the derivative in $\theta$ of the second term is

$$
\frac{k^{2} n^{4} \rho^{2} \sigma^{4}\left(6(\theta-1) \theta+n^{2}+1\right)+k n^{2} \rho \sigma^{2}\left(\theta^{2}(\theta(3 \theta+2)-3)+2 n^{4}+(1-3 \theta)^{2} n^{2}\right)+\left(\theta^{2}+n^{2}\right)^{3}}{k\left(\theta^{2}+n^{2}\left(k \rho \sigma^{2}+1\right)\right)^{3}} .
$$

We focus on the numerator and for part of the second term $\theta^{2}(\theta(3 \theta+2)-3)$, we can show that it reaches its minimum $-\frac{5}{16}$ when $\theta=1 / 2$, while our model assumption $n^{2} \geq 1 / 2$ implies $2 n^{4} \geq 1 / 2>\frac{5}{16}$; thus, the second term will be positive. Similarly, we can derive that part of the first term $6(\theta-1) \theta$ reaches its minimum $-3 / 2$ when $\theta=1 / 2$, while $n^{2}+1 \geq 3 / 2$, so this term is also positive. Thus, we can conclude that the derivative above is positive, so $\pi_{P}^{\mathrm{MAS}}$ is convex in $\theta$.

By computing the derivatives, we have

$$
\left.\frac{\partial \pi^{\mathrm{MAS}}}{\partial \theta}\right|_{\theta=0}=\left.\frac{\partial \pi^{\mathrm{MA}}}{\partial \theta}\right|_{\theta=0},\left.\quad \frac{\partial \pi^{\mathrm{MAS}}}{\partial \theta}\right|_{\theta=1}=\left.\frac{\partial \pi^{\mathrm{MA}}}{\partial \theta}\right|_{\theta=1} .
$$

Therefore, all the results for how $\pi^{\mathrm{MA}}$ varies with $\theta$ also apply here, and when $m$ and $n$ are relatively small, $\frac{\partial \pi^{\mathrm{MAS}}}{\partial \theta}$ will always be positive and $\pi^{\mathrm{MAS}}$ is increasing in $\theta$; otherwise, $\pi^{\mathrm{MAS}}$ can either be decreasing or decreasing first then increasing in $\theta$, which depends on the specific value of $m$.

For part (ii) of the proposition, note

$$
\begin{aligned}
& -\frac{\partial^{2} \pi^{\mathrm{MAS}}}{\partial n \partial m}=-\frac{2(\theta+1)^{4} k n^{3} \rho^{2} \sigma^{4}}{\left((\theta+1)^{2}+k m n^{2} \rho \sigma^{2}\right)^{3}}<0, \\
& -\frac{\partial^{2} \pi^{\mathrm{MAS}}}{\partial n \partial \theta}=\frac{4(\theta+1)^{3} k m n^{3} \rho^{2} \sigma^{4}}{\left((\theta+1)^{2}+k m n^{2} \rho \sigma^{2}\right)^{3}}+\frac{2(\theta-1) \theta n \rho \sigma^{2}\left(\theta^{2}+(2 \theta-1) n^{2}\left(k \rho \sigma^{2}+1\right)\right)}{\left(\theta^{2}+n^{2}\left(k \rho \sigma^{2}+1\right)\right)^{3}} .
\end{aligned}
$$

For the right-hand side of $-\frac{\partial^{2} \pi^{\mathrm{MAS}}}{\partial n \partial \theta}$, its first term is positive. Now, consider part of the numerator of the second term, $\theta^{2}+(2 \theta-1) n^{2}\left(k \rho \sigma^{2}+1\right)$, which is increasing in $\theta$ and must be positive when $\theta \geq 1 / 2$. Now, given $\theta<1 / 2$, this value is decreasing in $n, k, \rho, \sigma$, so when $n^{2}=1 / 2, k=\rho=\sigma=0$, it reaches its maximum and crosses 0 at $\theta=\frac{1}{2}(\sqrt{3}-1)$, so we can conclude that when $\theta<\frac{1}{2}(\sqrt{3}-1)$, this part must be negative, and therefore, the second term of $-\frac{\partial^{2} \pi^{\mathrm{MAS}}}{\partial n \partial \theta}$ is positive, as is the derivative. Otherwise, whether this derivative is positive or not depends on specific values of the model parameters.

Proof of Proposition 5.1. Denote the profit difference as

$$
\delta \pi=\pi^{\mathrm{MAS}}-\pi^{\mathrm{MA}}=\frac{(\theta-1)^{2} \theta^{2} \rho \sigma^{2}}{2\left(k \rho \sigma^{2}+1\right)\left(\theta^{2}+n^{2}\left(k \rho \sigma^{2}+1\right)\right)},
$$

and we have

$$
\begin{aligned}
\frac{\partial \delta \pi}{\partial n} & =-\frac{(\theta-1)^{2} \theta^{2} n \rho \sigma^{2}}{\left(\theta^{2}+n^{2}\left(k \rho \sigma^{2}+1\right)\right)^{2}}<0, \frac{\partial \delta \pi}{\partial \theta}=\frac{(\theta-1) \theta \rho \sigma^{2}\left(\theta^{3}+(2 \theta-1) n^{2}\left(k \rho \sigma^{2}+1\right)\right)}{\left(k \rho \sigma^{2}+1\right)\left(\theta^{2}+n^{2}\left(k \rho \sigma^{2}+1\right)\right)^{2}}, \\
\frac{\partial \delta \pi}{\partial k} & =-\frac{(\theta-1)^{2} \theta^{2} \rho^{2} \sigma^{4}\left(\theta^{2}+2 n^{2}\left(k \rho \sigma^{2}+1\right)\right)}{2\left(k \rho \sigma^{2}+1\right)^{2}\left(\theta^{2}+n^{2}\left(k \rho \sigma^{2}+1\right)\right)^{2}}<0, \\
\frac{\partial \delta \pi}{\partial \rho} & =\frac{(\theta-1)^{2} \theta^{2} \sigma^{2}\left(\theta^{2}+n^{2}\left(1-k^{2} \rho^{2} \sigma^{4}\right)\right)}{2\left(k \rho \sigma^{2}+1\right)^{2}\left(\theta^{2}+n^{2}\left(k \rho \sigma^{2}+1\right)\right)^{2}}, \frac{\partial \delta \pi}{\partial \sigma}=\frac{2 \rho}{\sigma} \frac{\partial \delta \pi}{\partial \rho} .
\end{aligned}
$$


Therefore, the monotonicity in $n$ and $k$ are shown. For $\frac{\partial \delta \pi}{\partial \theta}$, consider part of the numerator $\theta^{3}+(2 \theta-$ 1) $n^{2}\left(k \rho \sigma^{2}+1\right)$, which is increasing in $\theta$ with a negative value at $\theta=0$ and a positive value at $\theta=1$, so we can conclude that the derivative crosses 0 downward only once and the unimodality is shown. For $\frac{\partial \delta \pi}{\partial \rho}$, when $\rho$ increases (changing from 0 to $\infty$ ), its value will turn from positive to negative so the unimodality holds, and similar results can be shown for $\sigma$.

Now, with the properties derived above, first fixing other parameters and focusing on $\rho$ and $\sigma$, due to its unimodality, we find that $\delta \pi$ reaches its maximum when $\rho \sigma^{2}=\frac{\sqrt{\theta^{2}+n^{2}}}{n k}$, and the corresponding functional value is

$$
\frac{(\theta-1)^{2} \theta^{2} \sqrt{\theta^{2}+n^{2}}}{2 k\left(\sqrt{\theta^{2}+n^{2}}+n\right)\left(\theta^{2}+n\left(\sqrt{\theta^{2}+n^{2}}+n\right)\right)} .
$$

Given fixed $k$, this expression reaches its maximum at $n=1 / \sqrt{2}, \theta=\theta^{*}$, where $\theta^{*}$ satisfies

$$
\left(\theta^{*}\left(\sqrt{2\left(\theta^{*}\right)^{2}+1}-1\right)-1\right)\left(\theta^{*}\right)^{2}+\sqrt{2\left(\theta^{*}\right)^{2}+1}-1,
$$

and is the unique solution of its first-order condition in $\theta$. It can be numerically solved as $\theta^{*} \approx 0.457$. Therefore, the value of the maximum is approximately $0.013 / k$ by substituting the values of optimal $\theta$ and $n$.

Proof of Theorem 5.2. Denote $\delta \tilde{\pi}=\pi^{\mathrm{MA}}-\pi^{\mathrm{MT}}$

$$
\begin{aligned}
\delta \tilde{\pi}= & \frac{1}{2 k}\left(\frac{(\theta-1)^{2}}{k \rho \sigma^{2}+1}+\frac{(\theta+1)^{4}}{m\left((\theta+1)^{2}+k m n^{2} \rho \sigma^{2}\right)}\right) \\
& -\frac{(\theta+1)^{4}\left(k \rho \sigma^{2}+1\right)+(\theta-1)^{2} k m^{2} \rho \sigma^{2}\left(\theta^{2}+n^{2}\right)+(\theta-1)(\theta+1)^{2} m\left(\theta+2 \theta k \rho \sigma^{2}-1\right)}{2 k m\left((\theta+1)^{2}+k^{2} m n^{2} \rho^{2} \sigma^{4}+k \rho \sigma^{2}\left((\theta+1)^{2}+m\left(\theta^{2}+n^{2}\right)\right)\right)} \\
= & \frac{(\theta+1)^{4}\left(k \rho \sigma^{2}+1\right)+(\theta-1)^{2} k m^{2} n^{2} \rho \sigma^{2}+\left(\theta^{2}-1\right)^{2} m}{2 k m\left(k \rho \sigma^{2}+1\right)\left(\theta^{2}+2 \theta+k m n^{2} \rho \sigma^{2}+1\right)} \\
& -\frac{(\theta+1)^{4}\left(k \rho \sigma^{2}+1\right)+(\theta-1)^{2} k m^{2} \rho \sigma^{2}\left(\theta^{2}+n^{2}\right)+(\theta-1)(\theta+1)^{2} m\left(\theta+2 \theta k \rho \sigma^{2}-1\right)}{2 k m\left((\theta+1)^{2}+k^{2} m n^{2} \rho^{2} \sigma^{4}+k \rho \sigma^{2}\left((\theta+1)^{2}+m\left(\theta^{2}+n^{2}\right)\right)\right)} .
\end{aligned}
$$

By rearranging the numerators and denominators of two terms, we can show that $\delta \tilde{\pi}>0$ is equivalent to

$$
\begin{gathered}
2 \theta k^{2} m^{2} \rho \sigma^{2}\left(-(\theta-2)(\theta+1)^{4}-(\theta-1) k^{2} m n^{2} \rho^{2} \sigma^{4}(\theta(\theta(m+2)-m+4)+2)\right. \\
\left.-(\theta+1)^{2} k \rho \sigma^{2}\left(\theta^{3}-3 \theta+(\theta-1) m\left((\theta-1) \theta+2 n^{2}\right)-2\right)\right)>0 .
\end{gathered}
$$

Consider part of the left-hand side of the inequality

$$
\begin{aligned}
& -(\theta-2)(\theta+1)^{4}-(\theta-1) k^{2} m n^{2} \rho^{2} \sigma^{4}(\theta(\theta(m+2)-m+4)+2) \\
& -(\theta+1)^{2} k \rho \sigma^{2}\left(\theta^{3}-3 \theta+(\theta-1) m\left((\theta-1) \theta+2 n^{2}\right)-2\right),
\end{aligned}
$$

which is quadratic in $m$ and its second-order derivative in $m$ is $-2(\theta-1)^{2} \theta k^{2} n^{2} \rho^{2} \sigma^{4}<0$ implying the concavity in $m$, and we can solve the roots in $m$ :

$$
m_{1,2}=\frac{(\theta+1)^{2}\left(-\theta^{2}+\theta-2 n^{2}\left(k \rho \sigma^{2}+1\right) \pm \sqrt{(\theta-1)^{2} \theta^{2}+4 n^{4}\left(k \rho \sigma^{2}+1\right)^{2}+4 n^{2}\left(\theta+\theta k \rho \sigma^{2}\right)}\right)}{2(\theta-1) \theta k n^{2} \rho \sigma^{2}} .
$$

Consider $m_{1}$. From

$$
(\theta-1)^{2} \theta^{2}+4 n^{4}\left(k \rho \sigma^{2}+1\right)^{2}+4 n^{2}\left(\theta+\theta k \rho \sigma^{2}\right)-\left(-\theta^{2}+\theta-2 n^{2}\left(k \rho \sigma^{2}+1\right)\right)^{2}=4(2-\theta) \theta n^{2}\left(k \rho \sigma^{2}+1\right)>0,
$$


we learn

$$
-\theta^{2}+\theta-2 n^{2}\left(k \rho \sigma^{2}+1\right)<\sqrt{(\theta-1)^{2} \theta^{2}+4 n^{4}\left(k \rho \sigma^{2}+1\right)^{2}+4 n^{2}\left(\theta+\theta k \rho \sigma^{2}\right)} .
$$

Therefore, $m_{1}<0$ due to the negativity of its denominator. By comparing $m_{2}$ with $\bar{m}$, we have

$$
m_{2}-\bar{m}=\frac{(\theta+1)^{2}\left((\theta-1) \theta+\sqrt{(\theta-1)^{2} \theta^{2}+4 n^{4}\left(k \rho \sigma^{2}+1\right)^{2}+4 n^{2}\left(\theta+\theta k \rho \sigma^{2}\right)}\right)}{2(1-\theta) \theta k n^{2} \rho \sigma^{2}},
$$

but it is easy to find the right-hand side of the equation positive, so $m_{2}>\bar{m}$, and we can conclude that the focal expression above will always be positive when $m$ falls in its feasible region, which implies $\delta \tilde{\pi}>0$.

Proof of Corollary 5.3. To show $\beta_{1}^{\mathrm{MT}} \geq \beta_{1}^{\mathrm{MA}}$, i.e.,

$$
\beta_{1}^{\mathrm{MA}}-\beta_{1}^{\mathrm{MT}}=\frac{1-\theta}{k \rho \sigma^{2}+1}-\frac{(\theta+1)^{2}-(\theta-1) k m n^{2} \rho \sigma^{2}}{(\theta+1)^{2}+k^{2} m n^{2} \rho^{2} \sigma^{4}+k \rho \sigma^{2}\left((\theta+1)^{2}+m\left(\theta^{2}+n^{2}\right)\right)} \leq 0,
$$

it is equivalent to show

$$
\begin{aligned}
(1-\theta) & \left((\theta+1)^{2}+k^{2} m n^{2} \rho^{2} \sigma^{4}+k \rho \sigma^{2}\left((\theta+1)^{2}+m\left(\theta^{2}+n^{2}\right)\right)\right) \\
& -\left(k \rho \sigma^{2}+1\right)\left((\theta+1)^{2}-(\theta-1) k m n^{2} \rho \sigma^{2}\right) \\
= & -\theta(\theta+1)^{2}-\theta k \rho \sigma^{2}(\theta(\theta+(\theta-1) m+2)+1) \\
= & -\theta\left((\theta+1)^{2}+k^{2} m n^{2} \rho^{2} \sigma^{4}+k \rho \sigma^{2}\left((\theta+1)^{2}+m\left(\theta^{2}+n^{2}\right)\right)\right) \beta_{2}^{\mathrm{MT}} \leq 0,
\end{aligned}
$$

which obviously holds. To show $\beta_{2}^{\mathrm{MT}} \geq \beta_{2}^{\mathrm{MA}}$, i.e.,

$$
\beta_{2}^{\mathrm{MA}}-\beta_{2}^{\mathrm{MT}}=\frac{(\theta+1)^{2}}{(\theta+1)^{2}+k m n^{2} \rho \sigma^{2}}-\frac{(\theta+1)^{2}+k \rho \sigma^{2}(\theta(\theta+(\theta-1) m+2)+1)}{(\theta+1)^{2}+k^{2} m n^{2} \rho^{2} \sigma^{4}+k \rho \sigma^{2}\left((\theta+1)^{2}+m\left(\theta^{2}+n^{2}\right)\right)} \geq 0,
$$

it is equivalent to show

$$
\begin{aligned}
(\theta+1)^{2} & \left((\theta+1)^{2}+k^{2} m n^{2} \rho^{2} \sigma^{4}+k \rho \sigma^{2}\left((\theta+1)^{2}+m\left(\theta^{2}+n^{2}\right)\right)\right) \\
& -\left((\theta+1)^{2}+k \rho \sigma^{2}(\theta(\theta+(\theta-1) m+2)+1)\right)\left((\theta+1)^{2}+k m n^{2} \rho \sigma^{2}\right) \\
& =\theta k m \rho \sigma^{2}\left((\theta+1)^{2}+(1-\theta) k m n^{2} \rho \sigma^{2}\right) \geq 0,
\end{aligned}
$$

which obviously holds. Comparing $e_{i}^{\mathrm{MT}}$ and $e_{i}^{\mathrm{MA}}$, we have

$$
\begin{aligned}
& e_{1}^{\mathrm{MA}}-e_{1}^{\mathrm{MT}}=\frac{\theta \rho \sigma^{2}\left((\theta+1)^{2}+k \rho \sigma^{2}(\theta(\theta+(\theta-1) m+2)+1)\right)}{\left(k \rho \sigma^{2}+1\right)\left((\theta+1)^{2}+k^{2} m n^{2} \rho^{2} \sigma^{4}+k \rho \sigma^{2}\left((\theta+1)^{2}+m\left(\theta^{2}+n^{2}\right)\right)\right)}=\frac{\theta \rho \sigma^{2}}{k \rho \sigma^{2}+1} \beta_{2}^{\mathrm{MT}} \geq 0, \\
& e_{2}^{\mathrm{MA}}-e_{2}^{\mathrm{MT}}=\frac{\theta \rho \sigma^{2}\left((\theta+1)^{3}-\left(\theta^{2}-1\right) k m n^{2} \rho \sigma^{2}\right)}{\left((\theta+1)^{2}+k m n^{2} \rho \sigma^{2}\right)\left((\theta+1)^{2}+k^{2} m n^{2} \rho^{2} \sigma^{4}+k \rho \sigma^{2}\left((\theta+1)^{2}+m\left(\theta^{2}+n^{2}\right)\right)\right)} \geq 0 .
\end{aligned}
$$

Proof of Corollary 5.4. Substitute $e_{i}=e_{i}^{\mathrm{MA}}, i=1,2$ into the optimal response of the MSP under the multi-task case: $e_{1}=\frac{\beta_{1}-\beta_{2} \theta}{k}, e_{2}=\frac{\beta_{2}(\theta+1)}{m k}$, we can solve

$$
\tilde{\beta}_{1}^{\mathrm{MT}}=\frac{1-\theta}{k \rho \sigma^{2}+1}+\frac{\theta(\theta+1)^{2}}{(\theta+1)^{2}+k m n^{2} \rho \sigma^{2}}, \quad \tilde{\beta}_{2}^{\mathrm{MT}}=\frac{(\theta+1)^{2}}{(\theta+1)^{2}+k m n^{2} \rho \sigma^{2}} .
$$

Thus, we can compute

$$
\operatorname{RP}\left(\tilde{\beta}_{1}^{\mathrm{MT}}, \tilde{\beta}_{2}^{\mathrm{MT}}\right)=\frac{1}{2} \rho \sigma^{2}\left(\frac{(\theta+1)^{4} n^{2}}{\left((\theta+1)^{2}+k m n^{2} \rho \sigma^{2}\right)^{2}}+\left(\frac{1-\theta}{k \rho \sigma^{2}+1}+\frac{\theta(\theta+1)^{2}}{(\theta+1)^{2}+k m n^{2} \rho \sigma^{2}}\right)^{2}\right),
$$


while

$$
\operatorname{RP}\left(\beta_{1}^{\mathrm{MA}}, \beta_{2}^{\mathrm{MA}}\right)=\frac{1}{2} \rho \sigma^{2}\left(\frac{(\theta-1)^{2}}{\left(k \rho \sigma^{2}+1\right)^{2}}+\frac{(\theta+1)^{4} n^{2}}{\left((\theta+1)^{2}+k m n^{2} \rho \sigma^{2}\right)^{2}}\right)
$$

so the difference

$$
\operatorname{RP}\left(\tilde{\beta}_{1}^{\mathrm{MT}}, \tilde{\beta}_{2}^{\mathrm{MT}}\right)-\operatorname{RP}\left(\beta_{1}^{\mathrm{MA}}, \beta_{2}^{\mathrm{MA}}\right)=\frac{\theta(\theta+1)^{2} \rho \sigma^{2}\left(-\theta^{3}+3 \theta+k \rho \sigma^{2}\left(\theta(\theta+1)^{2}+2(1-\theta) m n^{2}\right)+2\right)}{2\left(k \rho \sigma^{2}+1\right)\left((\theta+1)^{2}+k m n^{2} \rho \sigma^{2}\right)^{2}}>0
$$

is shown since $0<\theta<1$.

Proof of Proposition 5.5. For the first part of the proposition, it suffices to show $-\frac{\partial \pi^{\mathrm{MA}}}{\partial n}>-\frac{\partial \pi^{\mathrm{MT}}}{\partial n}$ or $\frac{\partial \pi^{\mathrm{MA}}}{\partial n}<$ $\frac{\partial \pi^{\mathrm{MT}}}{\partial n}$. To show

$$
\begin{aligned}
\frac{\partial \pi^{\mathrm{MA}}}{\partial n}-\frac{\partial \pi^{\mathrm{MT}}}{\partial n} & =n \rho\left(-\frac{(\theta+1)^{4} \sigma^{2}}{\left((\theta+1)^{2}+k m n^{2} \rho \sigma^{2}\right)^{2}}+\frac{\left((\theta+1)^{2} \sigma+k \rho \sigma^{3}(\theta(\theta+(\theta-1) m+2)+1)\right)^{2}}{\left((\theta+1)^{2}+k^{2} m n^{2} \rho^{2} \sigma^{4}+k \rho \sigma^{2}\left((\theta+1)^{2}+m\left(\theta^{2}+n^{2}\right)\right)\right)^{2}}\right) \\
& <0,
\end{aligned}
$$

it is equivalent to show

$$
\begin{aligned}
\Delta= & (\theta+1)^{4} \sigma^{2}\left((\theta+1)^{2}+k^{2} m n^{2} \rho^{2} \sigma^{4}+k \rho \sigma^{2}\left((\theta+1)^{2}+m\left(\theta^{2}+n^{2}\right)\right)\right)^{2} \\
& \left((\theta+1)^{2} \sigma+k \rho \sigma^{3}(\theta(\theta+(\theta-1) m+2)+1)\right)^{2}\left(-\left((\theta+1)^{2}+k m n^{2} \rho \sigma^{2}\right)^{2}\right)>0,
\end{aligned}
$$

by rearranging denominators and numerators of the two fractions in the left-hand side of the inequality. We can introduce a monotone variable transform $x=n^{2}$, and $\Delta$ can be shown quadratic in $x$ with its second-order derivative in $x$ :

$$
2(1-\theta) \theta k^{3} m^{3} \rho^{3} \sigma^{8}\left(2(\theta+1)^{2}+k \rho \sigma^{2}(\theta(\theta(m+2)-m+4)+2)\right) .
$$

Note that the factor $2(\theta+1)^{2}+k \rho \sigma^{2}(\theta(\theta(m+2)-m+4)+2)$ of this derivative is decreasing in $m$ and its value is $(\theta+1)^{2}\left(k \rho \sigma^{2}+1\right)$ when $m=\bar{m}$; therefore, this factor must be positive in the feasible region of $m$, and thus, we can show the second-order derivative in $x$ is positive and $\Delta$ is convex in $x$.

Then, consider the value of $\Delta$ at $x=0$,

$$
\theta(\theta+1)^{4} k m \rho \sigma^{4}\left(2(\theta+1)^{2}+k \rho \sigma^{2}(\theta(2 \theta(m+1)-m+4)+2)\right) .
$$

Focus on the part $2(\theta+1)^{2}+k \rho \sigma^{2}(\theta(2 \theta(m+1)-m+4)+2)$, which is linear in $m$ with value $2(\theta+1)^{2}\left(k \rho \sigma^{2}+1\right)$ at $m=0$ and with value $\frac{(\theta+1)^{2}\left(k \rho \sigma^{2}+1\right)}{1-\theta}$ at $m=\bar{m}$, so both values are positive. Therefore, regardless of its monotonicity, we can conclude $\Delta>0$ at $x=0$.

Finally, consider

$$
\left.\frac{\partial \Delta}{\partial x}\right|_{x=0}=-2 \theta(\theta+1)^{2} k^{2} m^{2} \rho^{2} \sigma^{6}\left((\theta-2)(\theta+1)^{2}+k \rho \sigma^{2}\left(\theta\left(\theta^{2}+(\theta-1)^{2} m-3\right)-2\right)\right) .
$$

Again, focus on $(\theta-2)(\theta+1)^{2}+k \rho \sigma^{2}\left(\theta\left(\theta^{2}+(\theta-1)^{2} m-3\right)-2\right)$, which is linear in $m$ with value $(\theta-2)(\theta+$ $1)^{2}\left(k \rho \sigma^{2}+1\right)$ at $m=0$ and with value $-(\theta+1)^{2}\left(k \rho \sigma^{2}+1\right)$ at $m=\bar{m}$, so both values are negative. Therefore, for a similar reason as above, this derivative is positive.

Now, with all the properties (convexity, positivity of functional value and the value of the first-order derivative at $x=0$ ) derived above, we can conclude that $\Delta$ must be positive when $x>0$, thus, $\frac{\partial \pi^{\mathrm{MA}}}{\partial n}<\frac{\partial \pi^{\mathrm{MT}}}{\partial n}$ holds.

For the second part of the proposition, checking the values of $-\frac{\partial \pi^{i}}{\partial n}, i \in\{\mathrm{MT}, M A\}$ we find that all converge to 0 when $n \rightarrow \infty$, and the values of $\pi^{i}, i \in\{\mathrm{MT}, M A\}$ all converge to $\frac{(\theta-1)^{2}}{2 k\left(k \rho \sigma^{2}+1\right)}$ when $n \rightarrow \infty$. 
Proof of Proposition 5.6. From

$$
\frac{\partial e_{1}^{\mathrm{MT}}}{\partial n}=\frac{2 \theta k m n \rho^{2} \sigma^{4}}{(\theta+1)^{2}+k^{2} m n^{2} \rho^{2} \sigma^{4}+k \rho \sigma^{2}\left((\theta+1)^{2}+m\left(\theta^{2}+n^{2}\right)\right)} \beta_{2}^{\mathrm{MT}}>0, \quad \frac{\partial e_{1}^{\mathrm{MA}}}{\partial n}=0,
$$

we can derive the first inequality, and the limits can be derived with ease.

To show $\frac{\partial e_{2}^{\mathrm{MA}}}{\partial n}<\frac{\partial e_{2}^{\mathrm{MT}}}{\partial n}$, or

$$
2(\theta+1) n \rho \sigma^{2}\left(\frac{(\theta+1)^{2}}{\left((\theta+1)^{2}+k m n^{2} \rho \sigma^{2}\right)^{2}}-\frac{\left(k \rho \sigma^{2}+1\right)\left((\theta+1)^{2}+k \rho \sigma^{2}(\theta(\theta+(\theta-1) m+2)+1)\right)}{\left((\theta+1)^{2}+k^{2} m n^{2} \rho^{2} \sigma^{4}+k \rho \sigma^{2}\left((\theta+1)^{2}+m\left(\theta^{2}+n^{2}\right)\right)\right)^{2}}\right)>0,
$$

it is equivalent to show

$$
\begin{aligned}
\Delta= & (\theta+1)^{2}\left((\theta+1)^{2}+k^{2} m n^{2} \rho^{2} \sigma^{4}+k \rho \sigma^{2}\left((\theta+1)^{2}+m\left(\theta^{2}+n^{2}\right)\right)\right)^{2} \\
& -\left(k \rho \sigma^{2}+1\right)\left((\theta+1)^{2}+k \rho \sigma^{2}(\theta(\theta+(\theta-1) m+2)+1)\right)\left((\theta+1)^{2}+k m n^{2} \rho \sigma^{2}\right)^{2}>0
\end{aligned}
$$

Let $x=n^{2}$, we find

$$
\begin{aligned}
\left.\Delta\right|_{x=0} & =\theta(\theta+1)^{2} k m \rho \sigma^{2}\left((\theta+1)^{3}+k \rho \sigma^{2}(\theta(\theta(\theta+\theta m+3)+3)+1)\right)>0, \\
\left.\frac{\partial \Delta}{\partial x}\right|_{x=0} & =2 \theta(\theta+1)^{2} k^{2} m^{2} \rho^{2} \sigma^{4}\left(k \rho \sigma^{2}+1\right)>0,
\end{aligned}
$$

so $\Delta>0$ must hold. The limits can also be derived easily.

\section{Appendix B. Discussions on Model extensions}

Our paper constructs stylized models under the principal-agent framework, with assumptions such as that customer satisfaction is linear in effort levels ( $f_{1}$ and $f_{2}$ are linear functions), and that linear incentive plans are provided to agents. Although they facilitate the derivation of many analytical results, these assumptions may be strict to some extent. In this section, we consider the relaxation of some model assumptions, and discuss how it may influence our model results.

\section{B.1. Different parameters for the agents under the multi-agent scenario}

In Section 4, we analyze the multi-agent scenario, with the assumption that some of the model parameters (e.g., $\left.k_{1}, k_{2}\right)$ are the same as those in the multi-task scenario. In this subsection, we start a investigation on the case when these parameters can be different under the two scenarios.

Our focal parameters in this subsection are the marginal costs $\left(k_{1}, k_{2}\right)$. First note that all the results in Sections 3, 4, and Proposition 5.1 still hold since they are related to either model scenario and the differences in parameters do not affect these results. Now consider the other results in Section 5 . Let $k_{i}^{\mathrm{MT}}$ and $k_{i}^{\mathrm{MA}}(i=1,2)$ denote the cost coefficients under the two cases, respectively. We can find that if $k_{i}^{\mathrm{MT}} \geq k_{i}^{\mathrm{MA}}$ for $i=1,2$, most of the results in Theorem 5.1, Corollary 5.3, Propositions 5.5 and 5.6 will hold except for $\beta_{1}^{\mathrm{MA}} \leq \beta_{1}^{\mathrm{MT}}$. This is because that, $\beta_{i}^{\mathrm{MA}}, e_{i}^{\mathrm{MA}}$ and $\pi^{\mathrm{MA}}$ are decreasing in $m$ and $k$ from Proposition 4.2, while Theorem 5.2 shows that $\pi^{\mathrm{MA}}>\pi^{\mathrm{MT}}$ hold when $k_{i}^{\mathrm{MT}}=k_{i}^{\mathrm{MA}}$ (similar inequalities for $\beta_{2}$ and $e_{i}$ are also identified in Section 5). Thus when either $m$ or $k$ gets smaller under the multi-agent scenario, the inequalities above still hold. However, $\beta_{1}^{\mathrm{MA}} \leq \beta_{1}^{\mathrm{MT}}$ may not hold when $k_{i}^{\mathrm{MA}}$ get smaller than a threshold since $\beta_{1}^{\mathrm{MA}}$ is decreasing in $m$ and $k$. It is also not hard to prove the following inequalities:

$$
-\frac{\partial^{2} \pi^{\mathrm{MA}}}{\partial n \partial k} \leq 0,-\frac{\partial^{2} \pi^{\mathrm{MA}}}{\partial n \partial m} \leq 0,-\frac{\partial^{2} e_{i}^{\mathrm{MA}}}{\partial n \partial k} \leq 0,-\frac{\partial^{2} e_{i}^{\mathrm{MA}}}{\partial n \partial m} \leq 0 .
$$


Thus with a smaller $k_{i}^{\mathrm{MA}}$, the benefit from increasing CSI measure accuracy, i.e., $-\frac{\partial \pi^{\mathrm{MA}}}{\partial n}$ becomes larger, and the result validates Propositions 5.5 and 5.6.

All the results above show that, when delegating the two business lines to separate agents is more cost effective than to a multitasking agent, most of the analytical results in the main article are preserved. Note that this can be a reasonable assumption in some cases given that the professional service providers or business units are dedicated to a specific activity and work more efficiently. Now suppose $k_{i}^{\mathrm{MT}}<k_{i}^{\mathrm{MA}}$, there is the possibility that the conclusions discovered in Section 5 do not hold. We also consider the case where other parameters such as the magnitude of risk aversion $\rho$ may also vary for different scenarios or agents, and find the specific values of these parameters also determine the robustness of our model.

\section{B.2. Nonlinear formulations of the satisfaction function}

In this subsection, we will discuss an extended model with the two functions that compose customer satisfaction $S$, i.e., $f_{1}$ and $f_{2}$, being nonlinear in $\left(e_{1}, e_{2}\right)$. Following some existing literature $[2,35]$, we assume $f_{1}$ and $f_{2}$ are increasing and concave functions. Since analytical derivation of the optimal contracts and decisions are infeasible now, we mainly focus on numerical studies. Specifically, we let $f_{1}(x)=x^{c}(c \in(0,1))$, and assume $f_{2}(x)$ can have the following 3 possible formulations:

$$
\begin{aligned}
& f_{2}(x)=\theta\left(a x-b x^{2}\right)(\theta>0, a>0, b<0), \\
& f_{2}(x)=b \theta(\log (x+a)-\log (a))(a>0, b>0, x>-a), \\
& f_{2}(x)=\left\{\begin{array}{ll}
a \theta x & x<0 \\
b \theta x & x \geq 0
\end{array}(a>b>0) .\right.
\end{aligned}
$$

Note that the parameter $\theta$ is a magnitude factor similar to that when $f_{2}$ is linear, which affects the relative significance of disconfirmation in determining CS. The parameter settings of our numerical study under the base case are: $m=2, n=2, k=0.2, \rho=0.5, \sigma=1.5, \theta=1, c=0.5$; when $f_{2}$ is quadratic, $a=0.5, b=$ 0.025 ; when $f_{2}$ is logarithmic, $a=10, b=5$; when $f_{2}$ is piecewise linear, $a=0.6, b=0.4$. We also let all the parameters above except $a, b, c$ vary in specific ranges, respectively, and compute the corresponding optimal contract parameters, effort levels and the firm's profit. Then how these quantities change with different parameters can be compared with the analytical results such as the sensitivity analyses in Sections 3 and 4. Some of our findings are summarized as follows.

1. For Proposition 3.2, most of its results describing the changing patterns of $\beta_{i}^{\mathrm{MT}}$ and $\pi^{\mathrm{MT}}$ with model parameters can still be observed when $f_{2}$ is either a logarithmic or quadratic function, except for the impact of $m$ : $\beta_{i}^{\mathrm{MT}}(i=1,2)$ may not be monotone in $m$. When $f_{2}$ is a piecewise linear function, more discrepancies are observed between the numerical results and the monotone relationship in the proposition, and we find Part (iv) may not always hold.

2. For Proposition 4.2, most of its results still hold in the numerical study. For Proposition 4.4, some of its results such as the monotonicity of $\beta_{i}^{\mathrm{MAS}}$ and $e_{i}^{\mathrm{MAS}}$ in parameters like $n$ and $k$, may not hold. The discrepancies are observed for different formulations of $f_{2}$.

3. The relationship $\pi^{\mathrm{MAS}}>\pi^{\mathrm{MA}}$ in Proposition 5.1 naturally holds across all our tests, and $\pi^{\mathrm{MA}}>\pi^{\mathrm{MT}}$ in Theorem 5.2 holds in most of the cases. For Corollary 5.3, the inequalities on the relationship between $\beta_{i}^{\mathrm{MT}}$ and $\beta_{i}^{\mathrm{MA}}\left(e_{i}^{\mathrm{MT}}\right.$ and $\left.e_{i}^{\mathrm{MA}}\right)$ also hold in many computational instances (ove $85 \%$ among all instances). Proposition 5.5 holds in all cases in our test, and Proposition 5.6 holds in over $70 \%$ of all instances.

Note that the findings above are from a specific parameter setting. We also vary the values of parameters in the base case, and in general the observations are not much different from those listed above. Thus the nonlinearity $f_{1}$ and $f_{2}$ indeed has some influence on the behavior of optimal solutions qualitatively, while the robustness of our analytical findings also has its numerical validation to certain extents. 


\section{B.3. Double moral hazard}

In this subsection, we will initiate a preliminary analysis on the case when double moral hazard phenomenon exists in our model. This can happen when the firm is in charge of either the marketing or operational activity itself, mainly because it has some cost/efficiency advantages in conducting that business. For example, in $[10,16,17,55]$, the authors consider scenarios where a firm hiring salesforce to increase market demand and making inventory control decision himself; while Dong et al. [21] discuss the principal's involvement in direct selling and customer acquisition.

Most of the model specifications here are the same as those in the main article, and we assume the costs incurred due to certain activities is independent of the actual entity that carry out them, which is similar to the setting in [21]. Now first for the case where the principal exerts quality-control effort and the agent exerts sales effort, the corresponding incentive plan problem is as follows:

$$
\begin{aligned}
\max _{\alpha, \beta_{1}, \beta_{2}} & \pi=e_{1}^{*}+e_{2}^{*}+\theta\left(e_{2}^{*}-e_{1}^{*}\right)-\left(\alpha+\beta_{1} e_{1}^{*}+\beta_{2}\left(e_{2}^{*}+\theta\left(e_{2}^{*}-e_{1}^{*}\right)\right)\right) \\
\text { s.t. } & e_{1}^{*}=\underset{e_{1}}{\arg \max } \alpha+\beta_{1} e_{1}+\beta_{2}\left(e_{2}+\theta\left(e_{2}-e_{1}\right)\right)-\frac{1}{2} \rho\left(\beta_{1}^{2} \sigma^{2}+\beta_{2}^{2} \sigma_{m}^{2}\right)-\frac{1}{2} k_{1} e_{1}^{2}, \\
& e_{2}^{*}=\underset{e_{2}}{\arg \max } e_{1}+e_{2}+\theta\left(e_{2}-e_{1}\right)-\left(\alpha+\beta_{1} e_{1}+\beta_{2}\left(e_{2}+\theta\left(e_{2}-e_{1}\right)\right)\right)-\frac{1}{2} k_{2} e_{2}^{2}, \\
& \alpha+\beta_{1} e_{1}+\beta_{2}\left(e_{2}+\theta\left(e_{2}-e_{1}\right)\right)-\frac{1}{2} \rho\left(\beta_{1}^{2} \sigma^{2}+\beta_{2}^{2} \sigma_{m}^{2}\right)-\frac{1}{2} k_{1} e_{1}^{2} \geq 0 .
\end{aligned}
$$

Note that the principal incorporates CS into the agent's incentive plan. The first two constraints are the IC constraints for the agent and principal, respectively, and the third constraint is the agent's IR constraint. The optimal commission rates, effort levels and profit can be derived:

$$
\begin{aligned}
\beta_{1}^{\mathrm{DM} 1}= & \frac{(1-\theta)\left((\theta+1)^{2}+k m n^{2} \rho \sigma^{2}\right)}{(\theta+1)^{2}+k^{2} m n^{2} \rho^{2} \sigma^{4}+k \rho \sigma^{2}\left((\theta+1)^{2}+m\left(\theta^{2}+n^{2}\right)\right)}, \\
\beta_{2}^{\mathrm{DM} 1}= & \frac{(\theta-1) \theta k m \rho \sigma^{2}}{(\theta+1)^{2}+k^{2} m n^{2} \rho^{2} \sigma^{4}+k \rho \sigma^{2}\left((\theta+1)^{2}+m\left(\theta^{2}+n^{2}\right)\right)}, \\
e_{1}^{\mathrm{DM} 1}= & \frac{(1-\theta)\left((\theta+1)^{2}+k m \rho \sigma^{2}\left(\theta^{2}+n^{2}\right)\right)}{k\left((\theta+1)^{2}+k^{2} m n^{2} \rho^{2} \sigma^{4}+k \rho \sigma^{2}\left((\theta+1)^{2}+m\left(\theta^{2}+n^{2}\right)\right)\right)}, \\
e_{2}^{\mathrm{DM} 1}= & \frac{(\theta+1)\left((\theta+1)^{2}+k^{2} m n^{2} \rho^{2} \sigma^{4}+k \rho \sigma^{2}\left((\theta+1)^{2}+m\left(\theta+n^{2}\right)\right)\right)}{k m\left((\theta+1)^{2}+k^{2} m n^{2} \rho^{2} \sigma^{4}+k \rho \sigma^{2}\left((\theta+1)^{2}+m\left(\theta^{2}+n^{2}\right)\right)\right)}, \\
\pi^{\mathrm{DM} 1}= & \frac{(\theta+1)^{2} m\left((\theta-1)^{2}+k^{2} n^{2} \rho^{2} \sigma^{4}+k \rho \sigma^{2}\left(\theta^{2}+n^{2}\right)\right)}{2 k m\left((\theta+1)^{2}+k^{2} m n^{2} \rho^{2} \sigma^{4}+k \rho \sigma^{2}\left((\theta+1)^{2}+m\left(\theta^{2}+n^{2}\right)\right)\right)} \\
& +\frac{(\theta+1)^{4}\left(k \rho \sigma^{2}+1\right)+(\theta-1)^{2} k m^{2} \rho \sigma^{2}\left(\theta^{2}+n^{2}\right)}{2 k m\left((\theta+1)^{2}+k^{2} m n^{2} \rho^{2} \sigma^{4}+k \rho \sigma^{2}\left((\theta+1)^{2}+m\left(\theta^{2}+n^{2}\right)\right)\right)},
\end{aligned}
$$

where the superscript "DM1" denotes "double moral hazard (type 1)", i.e., the case when the principal carries out the quality-control effort. We can also conduct comparative static analyses for the optimal decisions.

Proposition B.1. (i) The effects of varying parameters on optimal decisions and profit are shown in Table B.1, where " $\uparrow, \downarrow,-, \sim "$ stand for increasing, decreasing, independent, and unclear, respectively.

(ii) The benefit of increasing CSI measure accuracy is more significant when the marginal cost of sales effort is relatively larger than the marginal cost of quality-control effort.

The proof of this proposition is similar to that of Proposition 3.2, which depends on the lengthy algebra after taking the corresponding partial derivatives. The detailed proof is omitted for brevity of exposition. We can 
TABLE B.1. Sensitivity analysis for the multi-task model under double moral hazard.

\begin{tabular}{lllllll}
\hline \hline & $m$ & $n$ & $\theta$ & $k$ & $\rho$ & $\sigma$ \\
\hline$\beta_{1}^{\text {DM1 }}$ & $\downarrow$ & $\uparrow$ & $\downarrow$ & $\downarrow$ & $\downarrow$ & $\downarrow$ \\
$\beta_{2}^{\text {DM1 }}$ & $\downarrow$ & $\uparrow$ & $\sim$ & $\sim$ & $\sim$ & $\sim$ \\
$e_{1}^{\text {DM1 }}$ & $\downarrow$ & $\uparrow$ & $\downarrow$ & $\downarrow$ & $\downarrow$ & $\downarrow$ \\
$e_{2}^{\text {DM1 }}$ & $\downarrow$ & $\downarrow$ & $\sim$ & $\downarrow$ & $\sim$ & $\sim$ \\
$\pi^{\text {DM1 }}$ & $\downarrow$ & $\downarrow$ & $\sim$ & $\downarrow$ & $\downarrow$ & $\downarrow$ \\
\hline
\end{tabular}

TABLE B.2. Sensitivity analysis for the multi-agent model under double moral hazard.

\begin{tabular}{lllllll}
\hline \hline & $m$ & $n$ & $\theta$ & $k$ & $\rho$ & $\sigma$ \\
\hline$\beta^{\text {DM2 }}$ & $\downarrow$ & $\downarrow$ & $\sim$ & $\downarrow$ & $\downarrow$ & $\downarrow$ \\
$e_{1}^{\text {DM2 }}$ & $\downarrow$ & $\downarrow$ & $\sim$ & $\downarrow$ & $\downarrow$ & $\downarrow$ \\
$e_{2}^{\text {DM2 }}$ & $\downarrow$ & $\downarrow$ & $\sim$ & $\downarrow$ & $\downarrow$ & $\downarrow$ \\
$\pi^{\text {DM2 }}$ & $\downarrow$ & $\downarrow$ & $\sim$ & $\downarrow$ & $\downarrow$ & $\downarrow$ \\
\hline
\end{tabular}

also build the incentive design for the case where the principal exerts sales effort as follows.

$$
\begin{array}{ll}
\max _{\alpha, \beta} & \pi=e_{1}^{*}+e_{2}^{*}+\theta\left(e_{2}^{*}-e_{1}^{*}\right)-\left(\alpha+\beta_{2}\left(e_{2}^{*}+\theta\left(e_{2}^{*}-e_{1}^{*}\right)\right)\right) \\
\text { s.t. } & e_{1}^{*}=\underset{e_{1}}{\arg \max } e_{1}+e_{2}+\theta\left(e_{2}-e_{1}\right)-\left(\alpha+\beta\left(e_{2}+\theta\left(e_{2}-e_{1}\right)\right)\right)-\frac{1}{2} k_{1} e_{1}^{2}, \\
& e_{2}^{*}=\underset{e_{2}}{\arg \max } \alpha+\beta\left(e_{2}+\theta\left(e_{2}-e_{1}\right)\right)-\frac{1}{2} \rho \beta^{2} \sigma_{m}^{2}-\frac{1}{2} k_{2} e_{2}^{2}, \\
& \alpha+\beta\left(e_{2}+\theta\left(e_{2}-e_{1}\right)\right)-\frac{1}{2} \rho \beta^{2} \sigma_{m}^{2}-\frac{1}{2} k_{2} e_{2}^{2} \geq 0 .
\end{array}
$$

The optimal contract parameter, effort levels and profit are as follows:

$$
\begin{aligned}
\beta^{\mathrm{DM} 2} & =\frac{(\theta+1)^{2}}{k m n^{2} \rho \sigma^{2}+\theta(\theta+\theta m+2)+1}, \\
e_{1}^{\mathrm{DM} 2} & =\frac{(\theta+1)^{3}}{k m\left(k m n^{2} \rho \sigma^{2}+\theta(\theta+\theta m+2)+1\right)}, \\
e_{2}^{\mathrm{DM} 2} & =\frac{\theta}{k}\left(\frac{(\theta+1)^{2}}{k m n^{2} \rho \sigma^{2}+\theta(\theta+\theta m+2)+1}-1\right)+\frac{1}{k}, \\
\pi^{\mathrm{DM} 2} & =\frac{(\theta-1)^{2}}{2 k}+\frac{(\theta+1)^{4}}{2 m k\left(k m n^{2} \rho \sigma^{2}+\theta(\theta+\theta m+2)+1\right)},
\end{aligned}
$$

where the superscript "DM2" denotes "double moral hazard (type 2)". Similar analyses on the characteristics of the optimal solutions can be derived.

Proposition B.2. (i) The effects of varying parameters on optimal decisions and profit are shown in Table B.2, where " $\uparrow, \downarrow,-, \sim "$ stand for increasing, decreasing, independent, and unclear, respectively.

(ii) The benefit of increasing CSI measure accuracy is more significant when the marginal cost of sales effort is relatively larger than the marginal cost of quality-control effort. 
Comparing the two propositions above with Propositions 4.2 and 4.4, we can find that both similarities and differences exist: Most of the quantities decrease with parameters $k, \rho, \sigma$; but the impacts of $\theta$ on both the optimal solutions and the benefit due to increasing CSI measure accuracy are not clear.

Finally we also compare the values of $\pi^{\mathrm{DM} 1}$ and $\pi^{\mathrm{DM} 2}$ to $\pi^{\mathrm{MA}}$ and $\pi^{\mathrm{MAS}}$, to explore whether results like Theorem 5.2 may hold. We discover that $\pi^{\mathrm{DM} 1}>\pi^{\mathrm{MAS}}$ holds, which implies that when the firm can carry out the quality-control activity itself with the same cost as the professional, then more profit can be generated. This result can be attributed to the less risk-averse of the principal, and is somewhat consistent with our argument in Section 5 that the decoupling of two activities may increase the firm's benefit.

To summarize, when double moral hazard issue is considered, some of the analytical results resemble those founded in our main article, whereas there are also differences between the two model. It should be mentioned that we have only conducted some simple and primary investigations on the robustness of our analytical results, and further studies may be necessary to give a deeper answer on this problem.

\section{B.4. Quota-bonus contracts}

We focus on incentive design with the optimal linear contracts mainly because it facilitates the closed-form solutions and that it is widely studied in many classical economics, marketing and operations literature. On the other hand, the quota-bonus contract [50] has increasing popularity in more recent literature $[10,16,17]$ and is intensively applied in business practice [12]. In this subsection we analyze the extension where the firm incentivizes the agent(s) with quota-bonus contracts. All the model settings are the same as those specified in the main article. Let $F_{1}, F_{2}$ denote the distribution functions of $\varepsilon_{1}, \varepsilon_{m}$, respectively.

For the multi-task scenario, note that the contract proposed in [50] is only base on a single performance measure, whereas in our model two performance measures $\left(D_{1}\right.$ and $\left.S\right)$ are included in the incentive plan. Thus for this case, we follow the corner compensation plan in [18], which consists of three parameters $\left(q_{1}, q_{2}, b\right)$, such that only when $D_{1} \geq q_{1}$ and $S \geq q_{2}$ will bonus $b$ be offered to the agent. The two-level optimization problem is formulated as follows:

$$
\begin{aligned}
& \max _{q_{1}, q_{2}, b} \pi=e_{1}^{*}+e_{2}^{*}+\theta\left(e_{2}^{*}-e_{1}^{*}\right)-U(b)\left(1-F_{1}\left(q_{1}-e_{1}^{*}\right)\right)\left(1-F_{2}\left(q_{2}-e_{2}^{*}-\theta\left(e_{2}^{*}-e_{1}^{*}\right)\right)\right) \\
& \text { s.t. } \quad e_{i}^{*}=\underset{e_{i}}{\arg \max } U(b)\left(1-F_{1}\left(q_{1}-e_{1}\right)\right)\left(1-F_{2}\left(q_{2}-e_{2}-\theta\left(e_{2}-e_{1}\right)\right)\right)-\frac{1}{2} k_{1} e_{1}^{2}-\frac{1}{2} k_{2} e_{2}^{2}, i=1,2 .
\end{aligned}
$$

Note that we use the relationships $\operatorname{Pr}\left\{q_{1} \geq D_{1}\right\}=1-F_{1}\left(q_{1}-e_{1}\right), \operatorname{Pr}\left\{q_{2} \geq S\right\}=1-F_{2}\left(q_{2}-e_{2}-\theta\left(e_{2}-e_{1}\right)\right)$.

In a similar manner, we can establish the counterpart model for the multi-agent scenario (for simplicity we only consider the model in Section 4.1 where the MSP is only incentivized with $D_{1}$ ). Now a quota-bonus contract with parameters $\left(q_{1}, b_{1}\right)$ is provided to the MSP and one with $\left(q_{2}, b_{2}\right)$ is provided to the OSP. The incentive design problem is formulated as below:

$$
\begin{aligned}
\max _{q_{1}, b_{1}, q_{2}, b_{2}} \pi & =e_{1}^{*}+e_{2}^{*}+\theta\left(e_{2}^{*}-e_{1}^{*}\right)-U\left(b_{1}\right)\left(1-F_{1}\left(q_{1}-e_{1}\right)\right)-U\left(b_{2}\right)\left(1-F_{2}\left(q_{2}-e_{2}-\theta\left(e_{2}-e_{1}\right)\right)\right) \\
\text { s.t. } \quad e_{1}^{*} & =\underset{e_{1}}{\arg \max } U\left(b_{1}\right)\left(1-F_{1}\left(q_{1}-e_{1}\right)\right)-\frac{1}{2} k_{1} e_{1}^{2}, \\
e_{2}^{*} & =\underset{e_{2}}{\arg \max } U\left(b_{2}\right)\left(1-F_{2}\left(q_{2}-e_{2}-\theta\left(e_{2}-e_{1}\right)\right)\right)-\frac{1}{2} k_{2} e_{2}^{2} .
\end{aligned}
$$

Note that for this problem, given the contracts provided by the firm, the two agents first determine their effort levels simultaneously. Then based on the agents' best responses, the firm then optimally adjust the contract parameters.

Unlike the analyses in the main article, we may only conduct numerical studies for this model when $F_{1}$ and $F_{2}$ are general distributions. We assume $\varepsilon_{1}$ and $\varepsilon_{m}$ follow normal distributions with the same parameters as those in Section 2, and the parameter settings are similar to those in Section B.2. In general, less trends or 
characteristics can be identified from our limited numerical results, compared with the monotone comparative statics derived in the main article. For example, both the optimal contract parameters and effort levels can be multimodal in cost/demand-related parameters. But we find that when the variance of outcomes (either $\sigma$ or $\sigma_{m}$ ) gradually get larger, both the efforts and the expected profit earned by the firm increase first then decrease. Another noticeable finding is that, unlike the case when linear contracts are applied, now the firm's profit under the multi-task scenario is frequently greater than that under the multi-agent scenario. The possible reason may be that, it is not easy for the agent to reach the targets on both performance measures even when a larger bonus is provided and higher effort levels are exerted, and the expected payment for the firm is relatively low under the multi-task scenario. While there is a higher possibility that either agent can earn the bonus, and the firm pays more to the service providers, which results in less profit.

\section{REFERENCES}

[1] S. Aflaki and I. Popescu, Managing retention in service relationships. Manage. Sci. 60 (2014) $415-433$.

[2] E.W. Anderson and M.W. Sullivan, The antecedents and consequences of customer satisfaction for firms. Market. Sci. 12 (1993) 125-143.

[3] A.K. Basu, R. Lal, V. Srinivasan and R. Staelin, Salesforce compensation plans: an agency theoretic perspective. Market. Sci. 4 (1985) 267-291.

[4] P. Bhardwaj, Delegating pricing decisions. Market. Sci. 20 (2001) 143-169.

[5] P. Bolton and M. Dewatripont, Contract Theory. MIT Press, Cambridge (2005).

[6] R.N. Bolton and J.H. Drew, A longitudinal analysis of the impact of service changes on customer attitudes. J. Market. 55 (1991) 1-9.

[7] L. Boyce, HSBC tells branch staff to improve customer satisfaction if they want bonuses as bank scraps sales incentives. Available at http://www.thisismoney.co.uk/money/saving/article-2281734/HSBC-scraps-staff-bonuseslinked-sales-targets.html (2013).

[8] F. Chen, Salesforce incentives, market information, and production/inventory planning. Manage. Sci. 51 (2005) 60-75.

[9] K. Chen, X. Wang, M. Huang and W.-K. Ching, Salesforce contract design, joint pricing and production planning with asymmetric overconfidence sales agent. J. Ind. Manage. Optim. 13 (2017) 873-899.

[10] L.Y. Chu and G. Lai, Salesforce contracting under demand censorship. Manuf. Serv. Oper. Manage. 15 (2013) $320-334$.

[11] W. Chu and P.S. Desai, Channel coordination mechanisms for customer satisfaction. Market. Sci. 14 (1995) $343-359$.

[12] D.J. Chung, T. Steenburgh and K. Sudhir, Do bonuses enhance sales productivity? A dynamic structural analysis of bonusbased compensation plans. Market. Sci. 33 (2013) 165-187.

[13] J.J. Cronin Jr, and S.A. Taylor, Measuring service quality: a reexamination and extension. J. Market. 56 (1992) 55-68.

[14] Y. Dai and X. Chao, Price delegation and salesforce contract design with asymmetric risk aversion coefficient of sales agents. Int. J. Prod. Econ. 172 (2016) 31-42.

[15] T. Dai and K. Jerath, Salesforce compensation with inventory considerations. Manage. Sci. 59 (2013) $2490-2501$.

[16] T. Dai and K. Jerath, Impact of inventory on quota-bonus contracts with rent sharing. Oper. Res. 64 (2016) 94-98.

[17] T. Dai and K. Jerath, Salesforce contracting under uncertain demand and supply: double moral hazard and optimality of smooth contracts. Working paper, Johns Hopkins University (2019).

[18] T. Dai, R. Ke and C. Ryan, Incentive design for operations-marketing multitasking. Working paper, Johns Hopkins University (2018).

[19] N. DeHoratius and A. Raman, Store manager incentive design and retail performance: an exploratory investigation. Manuf. Ser. Oper. Manage. 9 (2007) 518-534.

[20] S. Deng, T. Wang and X. Chang, Customer satisfaction incentives with budget constraints. Int. Trans. Oper. Res. 25 (2018) 1973-1995.

[21] Y. Dong, Y. Yao and T.H. Cui, When acquisition spoils retention: direct selling vs. delegation under CRM. Manage. Sci. 57 (2011) $1288-1299$.

[22] J. Feng, L. Liu and X. Liu, An optimal policy for joint dynamic price and lead-time quotation. Oper. Res. 59 (2011) $1523-1527$.

[23] Fidelity, Fidelity investments: introduction to representatives compensation. Available at https://www.fidelity.com/ bin-public/060_www_fidelity_com/documents/representative-compensation.pdf (2017).

[24] A. Hagiu and J. Wright, Controlling vs. enabling. Manage. Sci. 65 (2019) 577-595.

[25] S.M. Handley and J.V. Gray, Inter-organizational quality management: the use of contractual incentives and monitoring mechanisms with outsourced manufacturing. Prod. Oper. Manage. 22 (2013) 1540-1556.

[26] J.R. Hauser, D.I. Simester and B. Wernerfelt, Customer satisfaction incentives. Market. Sci. 13 (1994) $327-350$.

[27] T.H. Ho and Y.-S. Zheng, Setting customer expectation in service delivery: an integrated marketing-operations perspective. Manage. Sci. 50 (2004) 479-488.

[28] T.-H. Ho, Y.-H. Park and Y.-P. Zhou, Incorporating satisfaction into customer value analysis: optimal investment in lifetime value. Market. Sci. 25 (2006) 260-277. 
[29] Y.-C. Ho, J. Wu and Y. Tan, Disconfirmation effect on online rating behavior: a structural model. Inf. Syst. Res. 28 (2017) 626-642.

[30] B. Holmstrom and P. Milgrom, Aggregation and linearity in the provision of intertemporal incentives. Econ.: J. Econ. Soc. (1987) 303-328.

[31] B. Japsen, Ouch! patient satisfaction hits physician pay. Available at https://www.forbes.com/sites/brucejapsen/2013/07/ 02/patient-satisfaction-hits-physician-pay/\#1eb9e9ad54e5 (2013).

[32] K. Jerath, S. Netessine and Z.J. Zhang, Can we all get along? incentive contracts to bridge the marketing and operations divide. Working paper, Columbia University, New York (2007).

[33] A. Kalra, M. Shi and K. Srinivasan, Salesforce compensation scheme and consumer inferences. Manage. Sci. 49 (2003) 655-672.

[34] M. Kaya and Ö. Özer, Quality risk in outsourcing: Noncontractible product quality and private quality cost information. Nav. Res. Logist. (NRL) 56 (2009) 669-685.

[35] P.K. Kopalle and D.R. Lehmann, Setting quality expectations when entering a market: What should the promise be? Market. Sci. 25 (2006) 8-24.

[36] L.-C. Kung and Y.-J. Chen, The resource allocation problem for demand forecasting and performance measurement. Oper. Res. Lett. 40 (2012) 61-68.

[37] R. Lal and R. Staelin, Salesforce compensation plans in environments with asymmetric information. Market. Sci. 5 (1986) 179-198.

[38] R. Lal and V. Srinivasan, Compensation plans for single-and multi-product salesforces: an application of the holmstrom-milgrom model. Manage. Sci. 39 (1993) 777-793.

[39] C.-Y. Lee and R. Yang, Compensation plan for competing salespersons under asymmetric information. Eur. J. Oper. Res. 227 (2013) 570-580.

[40] D. Li, A brand that makes simple, aesthetically pleasing products became a sudden hit in China-here's how. Available at https://www.businessinsider.com/how-a-chinese-retail-site-became-popular-2017-9 (2017).

[41] L. Liu, M. Parlar and S.X. Zhu, Pricing and lead time decisions in decentralized supply chains. Manage. Sci. 53 (2007) 713-725.

[42] B. Liu, S. Ma, X. Guan and L. Xiao, Timing of sales commitment in a supply chain with manufacturer-quality and retailer-effort induced demand. Int. J. Prod. Econ. 195 (2018) 249-258.

[43] Luxeco, Turning "made in China" into a premium brand: China's internet giant netease has an ambitious plan for its private label. Available at http://en.luxe.co/post/190 (2017).

[44] P. Ma, H. Wang and J. Shang, Contract design for two-stage supply chain coordination: integrating manufacturer-quality and retailer-marketing efforts. Int. J. Prod. Econ. 146 (2013) 745-755.

[45] G.F. Mathewson and R.A. Winter, The economics of franchise contracts. J. Law Econ. 28 (1985) 503-526.

[46] B.K. Mishra and A. Prasad, Centralized pricing versus delegating pricing to the salesforce under information asymmetry. Market. Sci. 23 (2004) 21-27.

[47] S. Misra, A.T. Coughlan and C. Narasimhan, Salesforce compensation: an analytical and empirical examination of the agency theoretic approach. Quant. Market. Econ. 3 (2005) 5-39.

[48] M.E. Nikoofal and M. Gümüş, Quality at the source or at the end? Managing supplier quality under information asymmetry. Manuf. Serv. Oper. Manage. 20 (2018) 498-516.

[49] R.L. Oliver, Effect of expectation and disconfirmation on postexposure product evaluations: An alternative interpretation. $J$. Appl. Psychol. 62 (1977) 480.

[50] P. Oyer, A theory of sales quotas with limited liability and rent sharing. J. Labor Econ. 18 (2000) 405-426.

[51] P. Pekgün, P.M. Griffin and P. Keskinocak, Coordination of marketing and production for price and leadtime decisions. IIE Trans. 40 (2008) 12-30.

[52] E.L. Porteus, and S. Whang, On manufacturing/marketing incentives. Manage. Sci. 37 (1991) 1166-1181.

[53] D. Rouziès, V. Onyemah, et al. Sales force compensation: trends and research opportunities. Found. Trends® Market. 11 (2018) 143-214.

[54] O. Rubel and A. Prasad, Dynamic incentives in sales force compensation. Market. Sci. 35 (2015) 676-689.

[55] S. Saghafian and X. Chao, The impact of operational decisions on the design of salesforce incentives. Nav. Res. Logist. (NRL), 61 (2014) 320-340.

[56] A. Schöttner, Optimal sales force compensation in dynamic settings: commissions vs. bonuses. Manage. Sci. 63 (2016) 15291544 .

[57] B. Shen, Q. Li, C. Dong and V. Quan, Design outsourcing in the fashion supply chain: Oem versus odm. J. Oper. Res. Soc. 67 (2016) 259-268.

[58] K.C. So, Price and time competition for service delivery. Manuf. Serv. Oper. Manage. 2 (2000) 392-409.

[59] C.S. Tang, A review of marketing-operations interface models: from co-existence to coordination and collaboration. Int. J. Prod. Econ. 125 (2010) 22-40.

[60] N. Tepper, An Alibaba program lets small web retailers order small quantities from factories. Available at https://www. digitalcommerce360.com/2015/04/06/alibaba-b2b-program-lets-small-e-retailers-place-small-orders/ (2015).

[61] TMTPOST, Interview with GM of Xiaomi Youpin: the way Xiaomi does business of household items. Available at https://medium.com/@TMTpost/interview-with-gm-of-xiaomi-youpin-the-way-xiaomi-does-business-of-household-items62dc50fb333a (2018).

[62] Y. Wang, B. Niu and P. Guo, On the advantage of quantity leadership when outsourcing production to a competitive contract manufacturer. Prod. Oper. Manage. 22 (2013) 104-119. 
[63] T. Xiao, X. Cai and J. Jin, Pricing and effort investment decisions of a supply chain considering customer satisfaction. Int. J. Appl. Manage. Sci. 2 (2010) 1-19.

[64] G. Xie, S. Wang and K.K. Lai, Quality improvement in competing supply chains. Int. J. Prod. Econ. 134 (2011) $262-270$.

[65] S. Yang, X. Zhao, V. Shi, Y. Liao and J. Zhu, Joint determination of salesforce compensation, production, and pricing decisions. Int. J. Syst. Sci.: Oper. Logist. 2 (2015) 144-155. 